OPEN ACCESS

Edited by:

Benjamin Costas,

University of Porto, Portugal

Reviewed by:

Mansour Torfi Mozanzadeh,

South Iran Aquaculture Research

Center, Iran

Leonardo Julián Magnoni,

University of Porto, Portugal

*Correspondence.

Samad Rahimnejad srahimnejad@frov.jcu.cz

Specialty section: This article was submitted to Marine Fisheries, Aquaculture and Living Resources, a section of the journal

Frontiers in Marine Science

Received: 27 May 2021

Accepted: 05 July 2021

Published: 30 July 2021

Citation:

Rahimnejad S, Dabrowski K,

Izquierdo M, Malinovskyi O Kolářová J and Policar T (2021) Effects of Dietary Protein and Lipid Levels on Growth, Body Composition, Blood Biochemistry, Antioxidant Capacity and Ammonia Excretion of European Grayling (Thymallus thymallus). Front. Mar. Sci. 8:715636. doi: 10.3389/fmars.2021.715636

\section{Effects of Dietary Protein and Lipid Levels on Growth, Body Composition, Blood Biochemistry, Antioxidant Capacity and Ammonia Excretion of European Grayling (Thymallus thymallus)}

\author{
Samad Rahimnejad ${ }^{1 *}$, Konrad Dabrowski², Marisol Izquierdo3, Oleksandr Malinovskyi', \\ Jitka Kolářová ${ }^{1}$ and Tomas Policar ${ }^{1}$
}

${ }^{1}$ Faculty of Fisheries and Protection of Waters, South Bohemian Research Centre of Aquaculture and Biodiversity of Hydrocenoses, University of South Bohemia in České Budějovice, České Budějovice, Czechia, ${ }^{2}$ School of Environment and Natural Resources, The Ohio State University, Columbus, OH, United States, ${ }^{3}$ Aquaculture Research Group (GIA), Institute of Sustainable Aquaculture and Marine Ecosystems (ECOAQUA), Universidad de Las Palmas de Gran Canaria, Telde, Spain

This study evaluated growth, body composition, antioxidant capacity, innate immunity and ammonia excretion of European grayling (Thymallus thymallus) fed diets containing different protein and lipid contents. Six diets were produced to contain 30,40 , or $50 \%$ protein and 10 or $20 \%$ lipid. Juvenile fish averaging $25.2 \pm 0.28 \mathrm{~g}$ were stocked into eighteen 450-L circular tanks in a recirculating aquaculture system (RAS) and fed the test diets to satiation twice daily for 12 weeks. Fish weight gain (WG) was enhanced $(P<0.05)$ as dietary protein increased from 30\% (229\% WG) to 40\% (262\% WG) and plateaued thereafter. Enhancing protein and lipid content of diet led to reduced feed intake. Also, feed efficiency was improved by increasing dietary protein (by $40.8 \%$ ) and lipid (by 16.5\%) levels. An interaction of protein and lipid was found on whole-body lipid, and muscle lipid content increased as dietary lipid level increased. Muscle arachidonic acid (ARA), eicosapentaenoic acid (EPA, 20:5n-3) and total n-6 long-chain polyunsaturated fatty acids (LC-PUFA) contents enhanced by increasing dietary protein level. Moreover, increasing fat content of diet led to enhanced muscle linoleic acid, linolenic acid, total monounsaturated fatty acids (MUFA), total $n-6$, ratio of docosahexaenoic acid (DHA, 22:6n-3) to EPA and n-6/n-3. However, EPA, DHA, total n-6 LC-PUFA, total n-3, total n-3 LC-PUFA, and EPA/ARA ratio decreased at higher dietary lipid level. Serum triglyceride (TG) level and lactate dehydrogenase (LDH) activity decreased as dietary protein level increased. Increasing fat content of diet led to enhanced serum TG, cholesterol and glucose concentrations and reduced alanine aminotransferase, aspartate amino transferase and LDH activities. Serum malondialdehyde concentration was enhanced by increasing both dietary protein and lipid. Furthermore, serum myeloperoxidase activity was enhanced at higher dietary lipid 
level. Water ammonium nitrogen $\left(\mathrm{NH}_{4}{ }_{-}-\mathrm{N}\right)$ concentration was measured after 5 and $24 \mathrm{~h}$ of last feeding, and the results indicated the reduction of ammonia excretion as dietary lipid content increased. These findings suggest that $40 \%$ dietary protein can support optimal growth of juvenile European grayling reared in RAS and increasing lipid content from 10 to $20 \%$ can improve feed utilization and reduce ammonia excretion to the rearing water.

Keywords: feed efficiency, fatty acid profile, protein/energy ratio, Thymallus thymallus, weight gain

\section{INTRODUCTION}

Maximizing fish growth performance while reducing the production costs is the key to profitable aquaculture. Feed cost accounts for over $50 \%$ of the total expenses (Rahimnejad et al., 2015), and protein is the most costly feed ingredient particularly in carnivorous fish feed which should contain 40-60\% protein (NRC, 2011). Accordingly, determination of nutritional requirements of any new candidate species for aquaculture is necessary for formulation of a costeffective aquafeed.

Protein is not only essential for somatic growth but also required for tissues maintenance, and production of many key components such as hormones, enzymes and antibodies (Mir et al., 2020). Carnivorous fish including salmonids utilize protein as a source of energy preferentially to lipid and carbohydrate (Walton and Cowey, 1982), but its catabolism for energy production rather than being used for tissue synthesis increases the feed cost and leads to higher excretion of ammonia to the rearing water ( $\mathrm{Wu}$ and Gatlin, 2014) which adversely impacts fish feed consumption and growth (Kaushik and Medale, 1994). The efficient utilization of dietary protein not only relies on quantity and quality of dietary protein but also on sufficient supply of lipid and carbohydrate as energy sources (Ai et al., 2004). Considering the lower ability of carnivorous fish in metabolizing carbohydrates, lipids as energy-dense nutrient are better utilized as energy source by carnivorous fish (NRC, 1993). On the other hand, lipids play crucial roles in fish growth and health by providing essential fatty acids (Lee et al., 2002), and participating in uptake, transport and metabolism of fat-soluble vitamins and carotenoids (Jiang et al., 2015). It has been shown that adequate lipid supplementation in the feed can prohibit using protein for energy production (Lee et al., 2002). However, dietary lipid level could be increased up to a certain level beyond which undesirable effects could be attained such as lipid accumulation in the body and reduced growth performance resulting from the lack of essential nutrients due to reduced feed intake (Ali and Jauncey, 2005). It is believed that evaluation of the optimal ratio of dietary protein to energy is a more logic way of determining fish protein demand than quantifying merely the crude protein requirement (NRC, 2011) for production of efficient and environmentally friendly aquafeed.

European grayling (Thymallus thymallus) belongs to Salmonidae family which inhabits the waters of central, northern and north-eastern Europe. Its population has dwindled mainly due to the deterioration of its habitat, water contamination, and overfishing (Koskinen and Primmer, 2001; Uiblein et al., 2001; Carlstein, 2004; Susnik et al., 2004). Accordingly, conservation programs have been started to rehabilitate its stocks through releasing cultured fingerlings. In natural habitat it mainly feeds on small benthic organisms such as insects, crustaceans, mollusks and fish eggs, and large individuals can consume larvae of different fish species (Bolotov et al., 2012). However, the lack of information about its nutritional requirements remains as a major constraint to formulation of nutritionally adequate feed for this species. This study aimed to assess growth, body composition, blood biochemistry, antioxidant activity, immune response, and ammonia excretion of European grayling fed diets containing different protein and lipid levels.

\section{MATERIALS AND METHODS}

\section{Test Diets}

Six diets were prepared with varying protein (30, 40, and 50\%) and lipid (10 and 20\%) contents with protein to energy ratios ranging from 13.6 to $24 \mathrm{~g} \mathrm{MJ}^{-1}$ (Table 1). Fish meal and soy protein concentrate were used as the protein sources and a mixture of fish oil and soybean oil were used as the lipid sources. The experimental diets were prepared following the procedures described in our previous study (Rahimnejad et al., 2019). The dry ingredients were finely powdered and passed through a 250$\mu \mathrm{m}$ mesh, thoroughly mixed, and a dough was produced after addition of fish oil, soybean oil, lecithin, and water. Then, the pellets were produced in $3 \mathrm{~mm}$ diameter using a laboratory pelleting machine, crushed into desirable particle size, dried overnight in a ventilated dry oven at $35^{\circ} \mathrm{C}$, sealed in bags and stored at $-20^{\circ} \mathrm{C}$ until used.

\section{Fish Rearing}

Grayling larvae were obtained from local hatchery of Šumava Natural Park (Borová Lada, Czech Republic), and they were cultured for months in an experimental Recirculating Aquaculture System (RAS) under controlled conditions at Faculty of Fisheries and Protection of Waters, University of South Bohemia (FFWP, USB) (Vodnany, Czech Republic). Prior to starting the trial, the fish were moved and kept in 450-1 tanks in another RAS (FFWP, USB) for 4 weeks and fed a commercial diet (protein: $55 \%$, lipid: $16 \%$ ) to acclimatize them to the rearing conditions. Then, 1,800 fish of similar size (25.2 $\pm 0.28 \mathrm{~g})$ were distributed into eighteen 450-1 fiberglass circular tanks (100 fish/tank) containing $350 \mathrm{l}$ of freshwater and fed the test diets to 
TABLE 1 | Formulation and proximate composition of the experimental diets (\% dry matter).

\begin{tabular}{|c|c|c|c|c|c|c|}
\hline & P30L10 & P30L20 & P40L10 & P40L20 & P50L10 & P50L20 \\
\hline Fish meal ${ }^{\mathrm{a}}$ & 32.0 & 32.0 & 46.0 & 46.0 & 60.0 & 60.0 \\
\hline $\mathrm{SPC}^{\mathrm{b}}$ & 4.00 & 6.00 & 8.00 & 10.0 & 12.0 & 14.0 \\
\hline Wheat flour & 46.85 & 34.85 & 29.85 & 17.85 & 12.85 & 0.85 \\
\hline Brewer's yeast & 2.00 & 2.00 & 2.00 & 2.00 & 2.00 & 2.00 \\
\hline Dextrin & 4.00 & 4.00 & 4.00 & 4.00 & 4.00 & 4.00 \\
\hline Fish oil & 3.60 & 10.0 & 3.00 & 9.40 & 2.40 & 8.80 \\
\hline Soybean oil & 2.00 & 5.60 & 1.60 & 5.20 & 1.20 & 4.80 \\
\hline Lecithin & 1.00 & 1.00 & 1.00 & 1.00 & 1.00 & 1.00 \\
\hline Stay-C & 0.05 & 0.05 & 0.05 & 0.05 & 0.05 & 0.05 \\
\hline Mineral premix ${ }^{c}$ & 0.50 & 0.50 & 0.50 & 0.50 & 0.50 & 0.50 \\
\hline Vitamin premix & 0.50 & 0.50 & 0.50 & 0.50 & 0.50 & 0.50 \\
\hline Choline chloride & 0.50 & 0.50 & 0.50 & 0.50 & 0.50 & 0.50 \\
\hline Carboxymethylcellulose & 3.00 & 3.00 & 3.00 & 3.00 & 3.00 & 3.00 \\
\hline \multicolumn{7}{|l|}{ Proximate composition } \\
\hline Dry matter & 91.0 & 90.9 & 89.9 & 91.7 & 89.1 & 90.0 \\
\hline Protein & 29.8 & 30.3 & 40.0 & 40.3 & 49.2 & 49.7 \\
\hline Lipid & 9.90 & 19.0 & 9.70 & 19.3 & 10.1 & 19.5 \\
\hline Ash & 3.65 & 3.60 & 5.00 & 4.99 & 6.42 & 6.38 \\
\hline $\mathrm{GE}^{\mathrm{e}}$ & 20.2 & 22.3 & 20.4 & 22.4 & 20.5 & 22.5 \\
\hline P/E ratio ${ }^{f}$ & 14.8 & 13.6 & 19.6 & 18.0 & 24.0 & 22.1 \\
\hline
\end{tabular}

a Fish meal Super Prime (Crude protein: 67.9\%, crude fat: 9\%).

${ }^{b}$ Soy protein concentrate (Crude protein: $62 \%$, crude fat: $\left.0.5 \%\right)$.

${ }^{c}$ Mineral premix (mg or $g \mathrm{~kg}^{-1}$ diet): $\mathrm{NaF}, 2 \mathrm{mg} ; \mathrm{Kl}, 0.8 \mathrm{mg} ; \mathrm{CoCl}_{2} \cdot 6 \mathrm{H}_{2} \mathrm{O}$ (1\%), $50 \mathrm{mg}$; $\mathrm{CuSO}_{4} \cdot 5 \mathrm{H}_{2} \mathrm{O}, 10 \mathrm{mg} ; \mathrm{FeSO}_{4} \cdot \mathrm{H}_{2} \mathrm{O}, 80 \mathrm{mg} ; \mathrm{ZnSO} 4 \cdot \mathrm{H}_{2} \mathrm{O}, 50 \mathrm{mg}$; $\mathrm{MnSO} 4 \cdot \mathrm{H}_{2} \mathrm{O}, 25 \mathrm{mg} ; \mathrm{MgSO}_{4} \cdot 7 \mathrm{H}_{2} \mathrm{O}, 200 \mathrm{mg}$; zoelite, $4.582 \mathrm{~g}$.

'Vitamin premix (mg or $\mathrm{g} \mathrm{kg}^{-1}$ diet): thiamin, $10 \mathrm{mg}$; riboflavin, $8 \mathrm{mg}$; pyridoxine $\mathrm{HCl}, 10 \mathrm{mg}$; vitamin B12, $0.2 \mathrm{mg}$, vitamin K3, $10 \mathrm{mg}$; inositol, $100 \mathrm{mg}$; pantothenic acid, $20 \mathrm{mg}$; niacin acid, $50 \mathrm{mg}$; folic acid, $2 \mathrm{mg}$; biotin, $2 \mathrm{mg}$; retinol acetate, $400 \mathrm{mg}$; cholecalciferol, $5 \mathrm{mg}$; alpha-tocopherol, $100 \mathrm{mg}$; ethoxyquin, $150 \mathrm{mg}$; wheat middling, $1.1328 \mathrm{~g}$.

eCalculated gross energy (GE) ( $\left.\mathrm{MJ} \mathrm{kg}^{-1}\right)$ = based on combustion values of 23.6 MJ $\mathrm{kg}^{-1}$ for protein, $39.5 \mathrm{MJ} \mathrm{kg}{ }^{-1}$ for lipid and 17.2 $\mathrm{MJ} \mathrm{kg}^{-1}$ for carbohydrate.

${ }^{f} \mathrm{P} / \mathrm{E}$ ratio $\left(\mathrm{g} \mathrm{MJ}^{-1}\right)=$ Protein to energy ratio.

satiation twice daily for 12 weeks. Remaining feed in the tanks (if any) was siphoned out $30 \mathrm{~min}$ after feeding, dried and the data was used for calculation of total dry feed fed, and two-third of the water was replenished after the second feeding. Water temperature, $\mathrm{pH}$ and dissolved oxygen (DO) concentration were recorded daily during the experiment and their values were $16.5 \pm 0.03^{\circ} \mathrm{C}, 7.05 \pm 0.02$ and $11.7 \pm 0.05 \mathrm{mg} \mathrm{l}^{-1}$, respectively. Water nitrite $\left(\mathrm{NO}_{2}{ }^{-}\right)$and ammonium nitrogen $\left(\mathrm{NH}_{4}{ }^{+}-\mathrm{N}\right)$ concentrations were measured three times a week and estimated at $0.44 \pm 0.03 \mathrm{mg} \mathrm{l}^{-1}$ and $0.27 \pm 0.01 \mathrm{mg} \mathrm{l}^{-1}$, respectively. The photoperiod was kept at a 12:12 light/dark cycle.

\section{Sampling Protocol}

At the end of the feeding test, fish number and bulk weight were determined for estimation of survival rate and growth performance. Five intact fish from each tank were randomly captured, sacrificed with an overdose of 2-phenoxyethanol, and stored at $-20^{\circ} \mathrm{C}$ for proximate composition analysis. Also, dorsal muscle samples were collected from five fish per tank for proximate and fatty acid composition analyses. Blood was collected using 3-ml syringes from the caudal vein of six fish per tank after anesthetizing (200 $\mathrm{mg} \mathrm{l}^{-1}$ of 2-phenoxyethanol), transferred to 2-ml Eppendorf, and left to clot at $4^{\circ} \mathrm{C}$ for $24 \mathrm{~h}$. Then, the samples were centrifuged at $5,000 \times g$ for $10 \mathrm{~min}$ at $4^{\circ} \mathrm{C}$ and the serum was separated and stored at $-80^{\circ} \mathrm{C}$ for analyses of blood biochemical, antioxidant and innate immune parameters. Total length, individual weight, and viscera, liver and intraperitoneal fat weights of 10 fish from each tank were recorded to estimate Fulton's condition factor $(\mathrm{K})$, viscerosomatic index (VSI), hepatosomatic index (HSI), and intraperitoneal fat ratio (IPF). All the fish were fasted for $24 \mathrm{~h}$ before handling to reduce stress on fish.

\section{Analytical Methods Whole-Body and Muscle Composition}

Crude protein was measured based on the Kjeldahl technique (Asociation of Official Analytical Chemists (AOAC), 2002), crude lipid according to Folch et al. (1957), ash by combustion at $600^{\circ} \mathrm{C}$ (Asociation of Official Analytical Chemists (AOAC), 2002), and moisture content by drying at $110^{\circ} \mathrm{C}$ (Asociation of Official Analytical Chemists (AOAC), 2002). Fatty acid methyl esters of the lipids were obtained by transmethylation (Christie, 1989), which were subsequently separated by gas liquid chromatography as described earlier (Izquierdo et al., 1990).

\section{Blood Biochemistry, Immunity, and Antioxidant Activity}

Serum biochemical indices including total protein (TP), triglyceride (TG), total cholesterol (CHO), glucose (GLU), alanine aminotransferase (ALT), aspartate aminotransferase (AST), alkaline phosphatase (ALP), and lactate dehydrogenase (LDH) were measured using kits with a VET-TEST 8008 analyzer (IDEXX Laboratories Inc., Maine, United States). Superoxide dismutase (SOD) activity was determined using a SOD Assay Kit (Sigma, 19160). Commercial assay kits were used for measurement of catalase (CAT) activity (Thermo Fisher Scientific, CA, United States) and malondialdehyde (MDA) concentration (Sigma-Aldrich, Missouri, United States).

A turbidimetric method was used for determination of lysozyme activity according to Swain et al. (2007) with slight changes. Twenty microliters of serum was placed in flat-bottomed 96-well plates. Then, $180 \mu \mathrm{l}$ of Micrococcus lysodeikticus ( $0.2 \mathrm{mg} \mathrm{ml}^{-1}$, Sigma) suspension in $40 \mathrm{mM}$ sodium phosphate ( $\mathrm{pH}$ 6.2) was added as the lysozyme substrate to each well. Blank for each sample was prepared by adding $20 \mu \mathrm{l}$ of serum to $180 \mu \mathrm{l}$ of sodium phosphate buffer. The reduction in absorbance at $450 \mathrm{~nm}$ was measured after $20 \mathrm{~min}$ at $35^{\circ} \mathrm{C}$ in a BioRad 550 microplate reader. Absorbance reduction of $0.001 \mathrm{~min}^{-1}$ was considered as one unite of lysozyme activity.

Myeloperoxidase (MPO) activity was determined as described by Quade and Roth (1997). Shortly, $20 \mu$ l of serum was diluted with HBSS (Hanks Balanced Salt Solution) without $\mathrm{Ca}^{2+}$ or $\mathrm{Mg}^{2+}$ (Sigma-Aldrich, United States) in 96-well plates. Then, $35 \mu \mathrm{l}$ of $3,3^{\prime}, 5,5^{\prime}$-tetramethylbenzidine hydrochloride (TMB, $20 \mathrm{mM}$ ) (Sigma-Aldrich, United States) and $\mathrm{H}_{2} \mathrm{O}_{2}(5 \mathrm{mM})$ were added. The color change reaction was stopped after 2 min by adding $35 \mu \mathrm{l}$ of $4 \mathrm{M}$ sulfuric acid. Finally, the optical density was read at $450 \mathrm{~nm}$ in a microplate reader. 
Serum antiprotease activity was quantified based on Ellis et al. (1990) with some modifications (Magnadóttir et al., 1999). Twenty microliters of serum was incubated with $20 \mu \mathrm{l}$ of standard

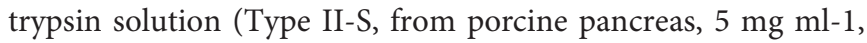
Sigma-Aldrich) for $10 \mathrm{~min}$ at $22^{\circ} \mathrm{C}$. Then, $200 \mu \mathrm{l}$ of phosphate buffer (0.1 M, pH 7.0) and $250 \mu \mathrm{l}$ azocasein (2\%) (Sigma) were added and incubated for $1 \mathrm{~h}$ at $22^{\circ} \mathrm{C}$. Thereafter, $500 \mu \mathrm{l}$ of trichloroacetic acid (10\%) was added and incubated for $30 \mathrm{~min}$ at $22^{\circ} \mathrm{C}$. Subsequently, the mixture was centrifuged at $6,000 \times \mathrm{g}$ for $5 \mathrm{~min}$ and $100 \mu \mathrm{l}$ of the supernatant was placed in the wells of $96-$ well flat-bottomed microplate containing $100 \mu \mathrm{l}$ of $1 \mathrm{~N} \mathrm{NaOH}$, and the OD was read at $430 \mathrm{~nm}$. For positive control, buffer replaced the serum, and for the negative control buffer replaced both serum and trypsin. The inhibition percentage of trypsin was estimated as follows:

$$
\text { Trypsin inhibition } \left.\%=\left(\mathrm{A}_{1}-\mathrm{A}_{2}\right) / \mathrm{A}_{1}\right) \times 100
$$

where $\mathrm{A}_{1}=$ control trypsin activity (without serum); $\mathrm{A}_{2}=$ trypsin activity remained after adding serum.

\section{Plasma Ammonia Concentration and Ammonia Excretion to Water}

To evaluate the effects of dietary treatments on plasma ammonia concentration, blood was collected from caudal vein of three fish from each of the main culturing tanks ( 9 fish per treatment) using heparinized syringes after $5 \mathrm{~h}$ of last feeding. Then plasma was separated by centrifugation at $5,000 \times g$ for $10 \mathrm{~min}$ at $4^{\circ} \mathrm{C}$ and kept at $-80^{\circ} \mathrm{C}$ until analysis. Plasma ammonia concentration was determined by the VET-TEST 8008 analyzer using a commercial kit.

To assess the effects of dietary treatments on fish ammonia excretion, 5 fish were randomly captured from each tank and moved to eighteen 40-1 glass aquaria containing 251 of freshwater. The fish were adapted to the new system for two days and fed with the same test diets to apparent satiation. After each feeding the remaining feed was removed and half of the water was replenished. On the $3 \mathrm{rd}$ day, the uneaten feed and fecal materials were syphoned out after feeding, water flow was stopped, and ammonium nitrogen $\left(\mathrm{NH}_{4}{ }^{+}-\mathrm{N}\right)$ concentration was measured after 5 and $24 \mathrm{~h}$ with a water analyzer (HANNA Instruments Inc., RI, United States) using kits. The following equation was used for the calculation of ammonia excretion (Rahimnejad and Lee, 2013):

$$
\text { Ammonia excretion }=\Delta \mathrm{N}-\mathrm{NH}_{4}{ }^{+} \times \mathrm{v} /(\mathrm{m} \times \mathrm{t})
$$

where $\Delta \mathrm{N}-\mathrm{NH}_{4}{ }^{+}$is the change in ammonium concentration during each test period; $\mathrm{v}$ is the water volume (l); $\mathrm{m}$ is the fish biomass $(\mathrm{kg})$; and $\mathrm{t}$ is the test period $(\mathrm{h})$.

Water temperature, $\mathrm{pH}$ and $\mathrm{DO}$ were measured at both sampling points and were evaluated to be $16.4 \pm 0.05^{\circ} \mathrm{C}$, $7.03 \pm 0.01$ and $8.02 \pm 0.12 \mathrm{mg} \mathrm{l}^{-1}$ at $5 \mathrm{~h}$, and $16.5 \pm 0.08^{\circ} \mathrm{C}$, $7.41 \pm 0.03$ and $8.82 \pm 0.04 \mathrm{mg} \mathrm{l}^{-1}$ at $24 \mathrm{~h}$ after last feeding.

\section{Statistical Analysis}

All the data are shown as mean \pm SE. Mean values of parameters were analyzed by one- and two-way ANOVAs to determine the significant differences due to the dietary levels of protein, lipid and their interaction. When ANOVA identified differences among groups, the difference in means was made with Tukey's test. Statistical significance was determined at $P<0.05$. All statistical analyses were carried out using Statistica version 13.5.0.17 (TIBCO Inc., CA, United States).

\section{RESULTS}

Growth of grayling juveniles was only responsive to dietary protein content $(P<0.05)$ but not to the fat content or their interaction (Table 2). As dietary protein level elevated from 30 to $40 \%$ growth performance enhanced significantly but no further improvement could be found at higher protein level. Feed intake (FI) decreased markedly as dietary protein and lipid contents increased. Feed efficiency (FE) was improved with enhancement of feed protein and lipid. The highest protein efficiency ratio (PER) was found at the $30 \%$ protein level and increasing dietary lipid content led to the remarkable enhancement of PER. No significant effect of dietary treatments could be found on fish survival rate which ranged from 98 to $100 \%$ (Table 2). Fulton's condition factor $(\mathrm{K})$ and HSI remained unaffected whereas VSI and IPF increased drastically by enhancing dietary fat content (Table 3).

A significant interaction of protein and lipid was found on whole-body lipid content. Moreover, enhancing lipid content of feed led to reduced whole-body moisture content (Table 4). Dorsal muscle lipid content increased by the increment of feed fat content, and muscle ash increased as dietary protein increased (Table 5). Fatty acid (FA) composition analysis revealed that oleic acid (OA) (18:1n-9), palmitic acid (16:0), linoleic acid (LA) (18:2n-6) and docosahexaenoic acid (DHA) (22:6n-3) were the dominant FAs in the dorsal muscle regardless of dietary treatments (Table 6). Increasing protein content of feed was accompanied with enhancement of $14: 0,14: 1 \mathrm{n}-7,15: 0,16: 0$, $16: 1 n-7,16: 2 n-4,17: 0,16: 4 n-3,18: 0,18: 1 n-7,18: 2 n-9,18: 2 n-$ $4,18: 4 n-1,20: 1 n-5,20: 2 n-9$, arachidonic acid (ARA) (20:4n$6)$, eicosapentaenoic acid (EPA) (20:5n-3), docosapentaenoic acid (22:5n-6), sum of saturated fatty acids and total n-6 long-chain polyunsaturated FA (LC-PUFA). However, sum of monounsaturated FAs (MUFA), total n-6, DHA/EPA and n-6/n-3 were decreased at higher protein levels. Muscle OA, LA, linolenic acid (LNA) (18:3n-3), 18:3n-1, 20:0, 20:1n-7, 20:2n-6, 20:3n-3, total MUFA and n- 6 contents, and ratios of DHA/EPA and n6/n-3 increased at higher dietary lipid level. Whilst decreased EPA, DHA, total n-6 LC-PUFA, total n-3, total n-3 LC-PUFA and EPA/ARA were found at higher dietary lipid level.

Increasing dietary protein level led to the reduction of serum TG concentration and enhancement of $\mathrm{CHO}$ concentration, and their values increased significantly at higher dietary lipid level. The lowest glucose level was found at $40 \%$ protein level which differed significantly from the other groups, and its value increased at higher lipid level. Serum ALT and AST activities were only affected by lipid level where lower activities were recorded at higher lipid level. Serum LDH activity decreased as feed protein and lipid contents increased. However, serum total protein level 
TABLE 2 | Growth, feed utilization and survival of European grayling (Thymallus thymallus) (25.2 $\pm 0.28 \mathrm{~g})$ fed the experimental diets for 12 weeks.

\begin{tabular}{|c|c|c|c|c|c|c|c|c|c|}
\hline Diets & Protein (\%) & Lipid (\%) & FBW & WG & SGR & $\mathbf{F I}$ & FE & PER & Survival (\%) \\
\hline \multicolumn{10}{|c|}{ Individual treatment means } \\
\hline P30L10 & 30 & 10 & 82.9 & 225 & 1.40 & 80.1 & 0.70 & 2.36 & 99.3 \\
\hline P40L10 & 40 & 10 & 85.6 & 243 & 1.47 & 69.9 & 0.85 & 2.13 & 98.0 \\
\hline P50L10 & 50 & 10 & 89.2 & 257 & 1.52 & 63.8 & 0.99 & 2.02 & 99.3 \\
\hline P30L20 & 30 & 20 & 82.5 & 232 & 1.43 & 69.1 & 0.82 & 2.72 & 98.7 \\
\hline P40L20 & 40 & 20 & 89.4 & 257 & 1.51 & 64.0 & 1.02 & 2.52 & 100 \\
\hline P50L20 & 50 & 20 & 92.5 & 266 & 1.54 & 59.7 & 1.15 & 2.31 & 100 \\
\hline SEM & & & 1.08 & 4.17 & 0.01 & 1.71 & 0.04 & 0.06 & 0.40 \\
\hline \multicolumn{10}{|c|}{ Means of main effects } \\
\hline & 30 & & $82.7^{b}$ & $229^{b}$ & $1.42^{b}$ & $74.6^{a}$ & $0.76^{c}$ & $2.54^{\mathrm{a}}$ & 99.0 \\
\hline & 40 & & $87.5^{\mathrm{a}}$ & $250^{a}$ & $1.49^{\mathrm{a}}$ & $66.9^{b}$ & $0.93^{b}$ & $2.32^{b}$ & 99.0 \\
\hline & 50 & & $90.8^{\mathrm{a}}$ & $262^{a}$ & $1.53^{\mathrm{a}}$ & $61.7^{c}$ & $1.07^{\mathrm{a}}$ & $2.17^{b}$ & 99.7 \\
\hline & & 10 & 85.9 & 242 & 1.46 & $71.3^{\mathrm{A}}$ & $0.85^{\mathrm{B}}$ & $2.17^{\mathrm{B}}$ & 98.9 \\
\hline & & 20 & 88.1 & 252 & 1.50 & $64.2^{\mathrm{B}}$ & $0.99^{A}$ & $2.52^{\mathrm{A}}$ & 99.6 \\
\hline \multicolumn{10}{|c|}{ Two-way ANOVA ( $P$-value) } \\
\hline Protein & & & 0.00 & 0.00 & 0.01 & 0.00 & 0.00 & 0.00 & 0.81 \\
\hline Lipid & & & 0.15 & 0.08 & 0.08 & 0.00 & 0.00 & 0.00 & 0.40 \\
\hline Interaction & & & 0.46 & 0.87 & 0.87 & 0.21 & 0.69 & 0.77 & 0.61 \\
\hline
\end{tabular}

Values are mean of triplicate groups $(n=3)$. Different superscript letters indicate significant difference among treatments $(P<0.05)$.

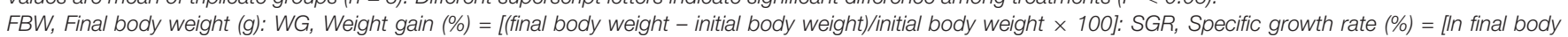

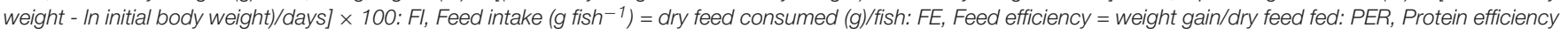
ratio $=$ wet weight gain/total protein given .

TABLE 3 | Organosomatic indices of European grayling (Thymallus thymallus) fed the experimental diets for 12 weeks.

\begin{tabular}{|c|c|c|c|c|c|c|}
\hline Diets & Protein $(\%)$ & Lipid (\%) & $\mathbf{K}$ & HSI & VSI & IPF \\
\hline & \multicolumn{6}{|c|}{ Individual treatment means } \\
\hline P30L10 & 30 & 10 & 1.07 & 0.84 & 10.7 & 2.42 \\
\hline P40L10 & 40 & 10 & 1.03 & 0.81 & 10.2 & 2.41 \\
\hline P50L10 & 50 & 10 & 1.04 & 0.78 & 9.65 & 2.07 \\
\hline P30L20 & 30 & 20 & 1.03 & 0.87 & 11.8 & 3.07 \\
\hline P40L20 & 40 & 20 & 1.04 & 0.81 & 11.4 & 3.68 \\
\hline P50L20 & 50 & 20 & 1.10 & 0.82 & 11.7 & 3.86 \\
\hline \multirow[t]{7}{*}{ SEM } & & & 0.01 & 0.02 & 0.22 & 0.19 \\
\hline & \multicolumn{6}{|c|}{ Means of main effects } \\
\hline & 30 & & 1.05 & 0.85 & 11.3 & 2.75 \\
\hline & 40 & & 1.03 & 0.81 & 10.8 & 3.05 \\
\hline & 50 & & 1.06 & 0.80 & 10.7 & 2.96 \\
\hline & & 10 & 1.04 & 0.81 & $10.1^{\mathrm{B}}$ & $2.30^{\mathrm{B}}$ \\
\hline & & 20 & 1.05 & 0.83 & $11.6^{\mathrm{A}}$ & $3.54^{\mathrm{A}}$ \\
\hline \multicolumn{7}{|c|}{ Two-way ANOVA (P-value) } \\
\hline Protein & & & 0.58 & 0.51 & 0.23 & 0.58 \\
\hline Lipid & & & 0.83 & 0.53 & 0.00 & 0.00 \\
\hline Interaction & & & 0.00 & 0.95 & 0.30 & 0.20 \\
\hline
\end{tabular}

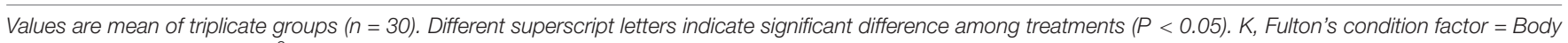

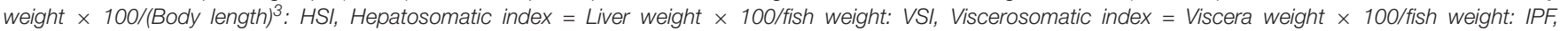
Intraperitoneal fat ratio $=$ Intraperitoneal fat weight $\times$ 100/fish weight.

and ALP activity did not vary among different experimental groups (Table 7).

Numerically higher serum SOD, MPO and antiprotease activities were found at $40 \%$ protein level although the differences were not statistically different. Serum MDA concentration was significantly enhanced by increment of lipid level and increasing protein content from 30 to 50\%. Moreover, increasing dietary lipid content led to the enhancement of serum MPO activity. No specific trends were observed for serum CAT and lysozyme activities (Table 8).

Water ammonium concentration after 5 and $24 \mathrm{~h}$ of last feeding was increased at higher protein levels while 
TABLE 4 | Whole-body proximate composition (\% dry weight) of European grayling (Thymallus thymallus) fed the experimental diets for 12 weeks.

\begin{tabular}{|c|c|c|c|c|c|c|}
\hline Diets & Protein (\%) & Lipid (\%) & Moisture & Protein & Lipid & Ash \\
\hline P30L10 & 30 & 10 & 72.0 & 59.1 & 29.0 & 11.1 \\
\hline P50L10 & 50 & 10 & 72.2 & 58.2 & 31.9 & 9.90 \\
\hline P3OL20 & 30 & 20 & 71.1 & 56.7 & 31.8 & 10.9 \\
\hline P4OL20 & 40 & 20 & 70.2 & 57.5 & 30.9 & 11.5 \\
\hline \multirow[t]{6}{*}{ SEM } & & & 0.23 & 0.34 & 0.41 & 0.26 \\
\hline & \multicolumn{6}{|c|}{ Means of main effects } \\
\hline & 30 & & 71.5 & 57.9 & 30.4 & 11.0 \\
\hline & 40 & & 71.0 & 58.1 & 30.0 & 11.9 \\
\hline & 50 & & 71.4 & 58.5 & 30.8 & 10.6 \\
\hline & & 10 & $72.0^{\mathrm{A}}$ & 58.7 & 30.0 & 11.1 \\
\hline Lipid & & & 0.00 & 0.16 & 0.29 & 0.72 \\
\hline Interaction & & & 0.65 & 0.17 & 0.03 & 0.17 \\
\hline
\end{tabular}

Values are mean of triplicate groups $(n=15)$. Different superscript letters indicate significant difference among treatments $(P<0.05)$.

TABLE 5 | Dorsal muscle proximate composition (\% dry weight basis) of European grayling (Thymallus thymallus) fed the experimental diets for 12 weeks.

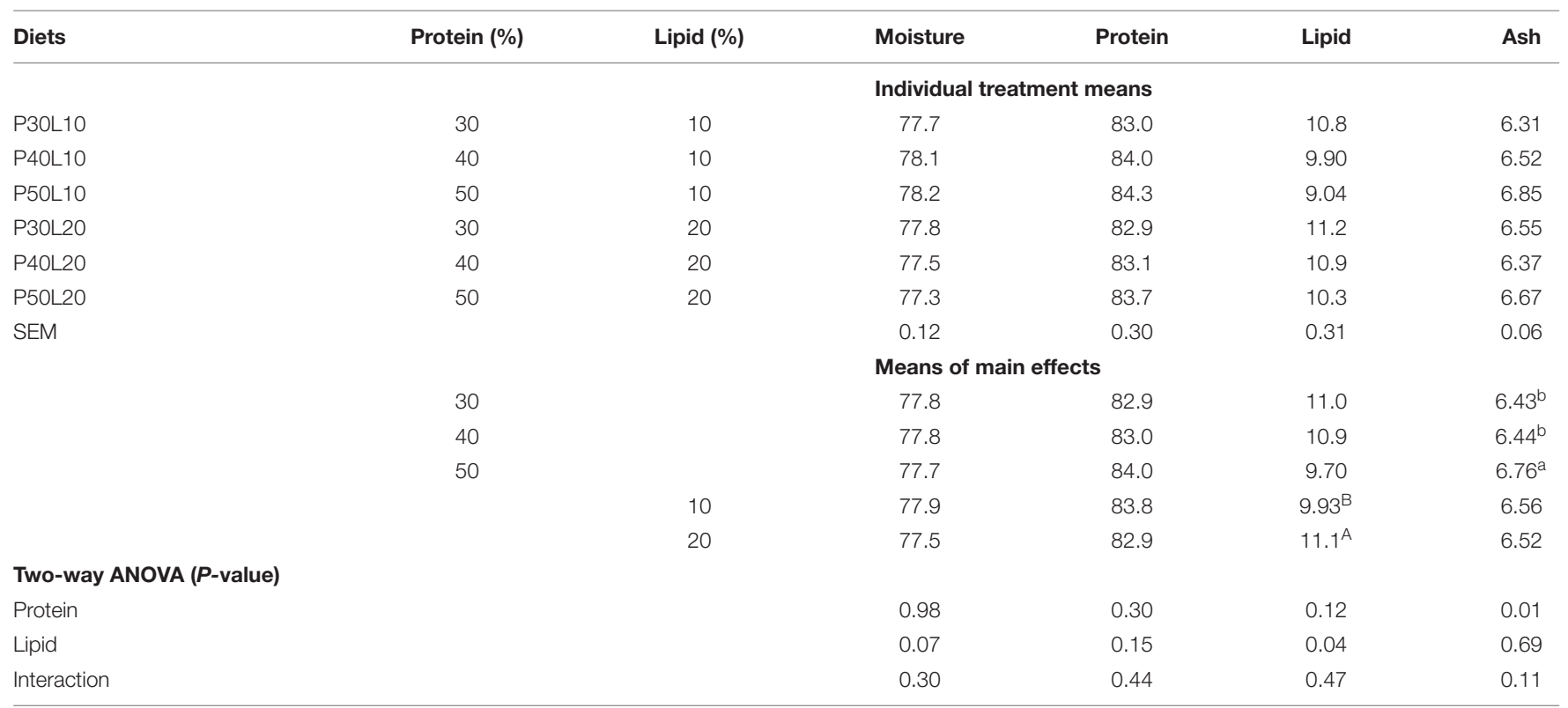

Values are mean of triplicate groups $(n=15)$. Different superscript letters indicate significant difference among treatments $(P<0.05)$.

increasing dietary lipid level reduced its concentration. Plasma ammonia concentration showed a similar trend to that of water ammonium concentration without significant difference among treatments (Table 9).

\section{DISCUSSION}

Protein requirement of various salmonid species ranges from 40 to $55 \%$ of diet (Halver and Hardy, 2002). Also, it has been shown that the desirable range of $\mathrm{P} / \mathrm{E}$ ratio for the most fish species is 17$26 \mathrm{~g} \mathrm{MJ}^{-1}$ (NRC, 1993). Lee and Kim (2001) examined the effects diets with varying protein $(30,40$, and $50 \%)$ and gross energy (GE) (19 and $21 \mathrm{MJ} \mathrm{kg}^{-1}$ ) contents on performance of masu salmon (Oncorhynchus masou Brevoort). Their results showed the significant improvement of fish growth by increasing dietary protein level from 30 to $40 \%$ while no further improvement was found at the higher protein level. Also, Xu et al. (2015) explored the impacts of diets containing different protein $(40$, 45,50 , and 55\%) and lipid (8 and 16\%) levels with P/E ratios 
TABLE 6 | Dorsal muscle fatty acid composition of European grayling (Thymallus thymallus) fed the experimental diets for 12 weeks (\% total identified FA).

\begin{tabular}{|c|c|c|c|c|c|c|c|c|c|c|c|c|c|c|c|}
\hline \multirow[t]{2}{*}{ Fatty acids } & \multicolumn{6}{|c|}{ Diets } & \multirow[t]{2}{*}{ SEM } & \multicolumn{3}{|c|}{ Protein (\%) } & \multicolumn{2}{|c|}{ Lipid (\%) } & \multicolumn{3}{|c|}{ Two-way ANOWA ( $P$-value) } \\
\hline & P30L10 & P30L20 & P40L10 & P40L20 & P50L10 & P50L20 & & 30 & 40 & 50 & 10 & 20 & Protein & Lipid & Interaction \\
\hline $14: 0$ & 1.99 & 1.82 & 1.99 & 2.02 & 2.67 & 2.03 & 0.08 & $1.91^{b}$ & $2.00^{\mathrm{b}}$ & $2.35^{\mathrm{a}}$ & $2.22^{\mathrm{A}}$ & $1.96^{\mathrm{B}}$ & 0.01 & 0.02 & 0.03 \\
\hline $14: 1 n-7$ & 0.02 & 0.01 & 0.03 & 0.02 & 0.04 & 0.02 & 0.00 & $0.01^{\mathrm{b}}$ & $0.02^{a b}$ & $0.03^{a}$ & $0.03^{\mathrm{A}}$ & $0.02^{\mathrm{B}}$ & 0.01 & 0.00 & 0.09 \\
\hline $14: 1 n-5$ & 0.06 & 0.06 & 0.06 & 0.07 & 0.07 & 0.06 & 0.00 & 0.06 & 0.06 & 0.07 & 0.06 & 0.06 & 0.13 & 0.69 & 0.08 \\
\hline $15: 0$ & 0.23 & 0.22 & 0.23 & 0.23 & 0.28 & 0.23 & 0.01 & $0.22^{b}$ & $0.23^{a b}$ & $0.25^{a}$ & $0.24^{\mathrm{A}}$ & $0.22^{\mathrm{B}}$ & 0.02 & 0.04 & 0.02 \\
\hline $15: 1 n-5$ & 0.01 & 0.01 & 0.01 & 0.01 & 0.01 & 0.01 & 0.00 & 0.01 & 0.01 & 0.01 & 0.01 & 0.01 & 0.97 & 0.09 & 0.42 \\
\hline 16:OISO & 0.03 & 0.03 & 0.03 & 0.04 & 0.04 & 0.03 & 0.00 & 0.03 & 0.04 & 0.04 & 0.03 & 0.03 & 0.15 & 0.69 & 0.41 \\
\hline $16: 0$ & 15.1 & 14.3 & 16.3 & 14.7 & 19.2 & 14.8 & 0.45 & $14.7^{\mathrm{b}}$ & $15.5^{\mathrm{b}}$ & $17.0^{\mathrm{a}}$ & $16.9^{\mathrm{A}}$ & $14.6^{\mathrm{B}}$ & 0.01 & 0.00 & 0.01 \\
\hline $16: 1 n-7$ & 3.39 & 2.81 & 3.88 & 3.17 & 4.65 & 3.15 & 0.15 & $3.10^{\mathrm{c}}$ & $3.53^{b}$ & $3.90^{a}$ & $3.97^{\mathrm{A}}$ & $3.04^{\mathrm{B}}$ & 0.00 & 0.00 & 0.00 \\
\hline $16: 1 n-5$ & 0.10 & 0.08 & 0.09 & 0.09 & 0.11 & 0.09 & 0.00 & 0.09 & 0.09 & 0.10 & $0.10^{\mathrm{A}}$ & $0.08^{\mathrm{B}}$ & 0.05 & 0.02 & 0.17 \\
\hline $16: 2 n-6$ & 0.00 & 0.00 & 0.01 & 0.01 & 0.00 & 0.01 & 0.00 & 0.00 & 0.01 & 0.01 & 0.01 & 0.01 & 0.07 & 0.90 & 0.69 \\
\hline $16: 2 n-4$ & 0.21 & 0.17 & 0.23 & 0.20 & 0.26 & 0.21 & 0.01 & $0.19^{c}$ & $0.22^{b}$ & $0.24^{\mathrm{a}}$ & $0.23^{A}$ & $0.20^{\mathrm{B}}$ & 0.00 & 0.00 & 0.12 \\
\hline $17: 0$ & 0.18 & 0.13 & 0.20 & 0.16 & 0.23 & 0.18 & 0.01 & $0.16^{c}$ & $0.18^{b}$ & $0.20^{a}$ & $0.20^{\mathrm{A}}$ & $0.16^{\mathrm{B}}$ & 0.00 & 0.00 & 0.22 \\
\hline $16: 3 n-4$ & 0.18 & 0.18 & 0.18 & 0.19 & 0.21 & 0.18 & 0.00 & 0.18 & 0.18 & 0.19 & 0.19 & 0.18 & 0.11 & 0.09 & 0.06 \\
\hline $16: 3 n-3$ & 0.10 & 0.08 & 0.11 & 0.08 & 0.09 & 0.08 & 0.01 & 0.09 & 0.10 & 0.09 & 0.10 & 0.08 & 0.78 & 0.10 & 0.72 \\
\hline $16: 3 n-1$ & 0.02 & 0.02 & 0.03 & 0.02 & 0.03 & 0.02 & 0.00 & 0.02 & 0.02 & 0.02 & $0.03^{A}$ & $0.02^{B}$ & 0.15 & 0.00 & 0.10 \\
\hline $16: 4 n-3$ & 0.15 & 0.10 & 0.18 & 0.13 & 0.19 & 0.15 & 0.01 & $0.13^{b}$ & $0.15^{\mathrm{a}}$ & $0.17^{a}$ & $0.17^{A}$ & $0.13^{\mathrm{B}}$ & 0.01 & 0.00 & 0.84 \\
\hline $16: 4 n-1$ & 0.02 & 0.01 & 0.03 & 0.01 & 0.02 & 0.02 & 0.00 & 0.02 & 0.02 & 0.02 & $0.02^{\mathrm{A}}$ & $0.01^{B}$ & 0.80 & 0.01 & 0.35 \\
\hline 18:0 & 3.31 & 3.04 & 3.65 & 3.16 & 4.07 & 3.28 & 0.09 & $3.17^{b}$ & $3.40^{\mathrm{b}}$ & $3.68^{a}$ & $3.68^{\mathrm{A}}$ & $3.16^{\mathrm{B}}$ & 0.00 & 0.00 & 0.06 \\
\hline $18: 1 n-9$ & 27.6 & 34.7 & 24.8 & 33.6 & 23.4 & 30.6 & 1.05 & $31.2^{\mathrm{a}}$ & $29.2^{a}$ & $27.0^{c}$ & $25.3^{\mathrm{B}}$ & $32.9^{\mathrm{A}}$ & 0.00 & 0.00 & 0.42 \\
\hline $18: 1 n-7$ & 2.83 & 2.67 & 2.96 & 2.80 & 3.20 & 2.80 & 0.05 & $2.75^{\mathrm{b}}$ & $2.88^{a}$ & $3.00^{\mathrm{a}}$ & $3.00^{\mathrm{A}}$ & $2.76^{\mathrm{B}}$ & 0.03 & 0.00 & 0.27 \\
\hline $18: 1 n-5$ & 0.13 & 0.12 & 0.12 & 0.12 & 0.13 & 0.12 & 0.00 & 0.13 & 0.12 & 0.12 & 0.12 & 0.12 & 0.46 & 0.57 & 0.11 \\
\hline $18: 2 n-9$ & 0.06 & 0.05 & 0.06 & 0.06 & 0.07 & 0.05 & 0.00 & $0.05^{b}$ & $0.06^{a}$ & $0.06^{a}$ & $0.07^{A}$ & $0.05^{\mathrm{B}}$ & 0.00 & 0.00 & 0.07 \\
\hline $18: 2 n-6$ & 10.8 & 11.7 & 8.65 & 10.7 & 7.03 & 9.56 & 0.38 & $11.2^{\mathrm{a}}$ & $9.70^{b}$ & $8.30^{c}$ & $8.81^{\mathrm{B}}$ & $10.7^{A}$ & 0.00 & 0.00 & 0.00 \\
\hline $18: 2 n-4$ & 0.14 & 0.11 & 0.16 & 0.13 & 0.19 & 0.14 & 0.01 & $0.13^{\mathrm{c}}$ & $0.15^{c}$ & $0.17^{a}$ & $0.17^{\mathrm{A}}$ & $0.13^{\mathrm{B}}$ & 0.00 & 0.00 & 0.00 \\
\hline $18: 3 n-6$ & 0.24 & 0.22 & 0.18 & 0.19 & 0.15 & 0.18 & 0.01 & $0.23^{a}$ & $0.19^{b}$ & $0.18^{b}$ & 0.19 & 0.20 & 0.00 & 0.38 & 0.14 \\
\hline $18: 3 n-4$ & 0.13 & 0.10 & 0.13 & 0.11 & 0.13 & 0.12 & 0.00 & 0.11 & 0.12 & 0.13 & $0.13^{\mathrm{A}}$ & $0.11^{\mathrm{B}}$ & 0.19 & 0.00 & 0.45 \\
\hline $18: 3 n-3$ & 2.33 & 2.89 & 1.96 & 2.81 & 1.58 & 2.61 & 0.11 & $2.61^{a}$ & $2.39^{b}$ & $2.10^{c}$ & $1.96^{\mathrm{B}}$ & $2.77^{\mathrm{A}}$ & 0.00 & 0.00 & 0.00 \\
\hline $18: 3 n-1$ & 0.02 & 0.03 & 0.02 & 0.03 & 0.02 & 0.03 & 0.00 & 0.03 & 0.03 & 0.02 & $0.02^{\mathrm{B}}$ & $0.03^{A}$ & 0.84 & 0.00 & 0.93 \\
\hline $18: 4 n-3$ & 0.80 & 0.69 & 0.76 & 0.75 & 0.72 & 0.76 & 0.02 & 0.74 & 0.75 & 0.74 & 0.76 & 0.73 & 0.91 & 0.38 & 0.16 \\
\hline $18: 4 n-1$ & 0.11 & 0.08 & 0.13 & 0.10 & 0.13 & 0.11 & 0.00 & $0.10^{b}$ & $0.11^{a}$ & $0.12^{a}$ & $0.12^{\mathrm{A}}$ & $0.10^{\mathrm{B}}$ & 0.00 & 0.00 & 0.69 \\
\hline $20: 0$ & 0.18 & 0.21 & 0.17 & 0.20 & 0.17 & 0.19 & 0.00 & 0.19 & 0.19 & 0.18 & $0.17^{\mathrm{B}}$ & $0.20^{\mathrm{A}}$ & 0.22 & 0.00 & 0.94 \\
\hline $20: 1 n-9$ & 0.32 & 0.30 & 0.30 & 0.31 & 0.30 & 0.30 & 0.01 & 0.31 & 0.30 & 0.30 & 0.31 & 0.30 & 0.60 & 0.82 & 0.48 \\
\hline $20: 1 n-7$ & 2.81 & 2.88 & 2.48 & 2.79 & 2.45 & 2.68 & 0.05 & $2.84^{\mathrm{a}}$ & $2.64^{\mathrm{ab}}$ & $2.57^{b}$ & $2.58^{\mathrm{B}}$ & $2.78^{\mathrm{A}}$ & 0.02 & 0.01 & 0.36 \\
\hline $20: 1 n-5$ & 0.17 & 0.16 & 0.17 & 0.16 & 0.19 & 0.17 & 0.00 & $0.16^{b}$ & $0.17^{a b}$ & $0.19^{a}$ & $0.18^{A}$ & $0.16^{\mathrm{B}}$ & 0.03 & 0.00 & 0.49 \\
\hline $20: 2 n-9$ & 0.07 & 0.05 & 0.08 & 0.07 & 0.09 & 0.07 & 0.00 & $0.06^{b}$ & $0.08^{a b}$ & $0.08^{a}$ & $0.08^{A}$ & $0.07^{\mathrm{B}}$ & 0.03 & 0.00 & 0.94 \\
\hline $20: 2 n-6$ & 0.62 & 0.62 & 0.55 & 0.60 & 0.46 & 0.58 & 0.01 & $0.62^{a}$ & $0.58^{\mathrm{b}}$ & $0.52^{c}$ & $0.54^{\mathrm{B}}$ & $0.60^{A}$ & 0.00 & 0.00 & 0.00 \\
\hline $20: 3 n-9$ & 0.04 & 0.03 & 0.03 & 0.03 & 0.04 & 0.03 & 0.00 & 0.03 & 0.03 & 0.04 & $0.04^{\mathrm{A}}$ & $0.03^{B}$ & 0.18 & 0.01 & 0.11 \\
\hline $20: 3 n-6$ & 0.24 & 0.17 & 0.23 & 0.18 & 0.19 & 0.17 & 0.01 & $0.20^{a}$ & $0.20^{a}$ & $0.18^{b}$ & $0.22^{\mathrm{A}}$ & $0.17^{\mathrm{B}}$ & 0.00 & 0.00 & 0.00 \\
\hline $20: 4 n-6$ & 0.67 & 0.49 & 0.81 & 0.55 & 0.86 & 0.65 & 0.03 & $0.58^{\mathrm{c}}$ & $0.68^{b}$ & $0.76^{a}$ & $0.78^{A}$ & $0.56^{\mathrm{B}}$ & 0.00 & 0.00 & 0.23 \\
\hline $20: 3 n-3$ & 0.21 & 0.24 & 0.19 & 0.24 & 0.17 & 0.23 & 0.01 & $0.23^{a}$ & $0.22^{a b}$ & $0.20^{b}$ & $0.19^{\mathrm{B}}$ & $0.24^{A}$ & 0.01 & 0.00 & 0.06 \\
\hline $20: 4 n-3$ & 0.44 & 0.40 & 0.45 & 0.42 & 0.43 & 0.46 & 0.01 & 0.42 & 0.44 & 0.44 & 0.44 & 0.42 & 0.44 & 0.28 & 0.09 \\
\hline $20: 5 n-3$ & 4.24 & 2.69 & 5.01 & 3.21 & 5.24 & 3.85 & 0.23 & $3.46^{b}$ & $4.11^{a}$ & $4.55^{a}$ & $4.83^{\mathrm{A}}$ & $3.25^{\mathrm{B}}$ & 0.00 & 0.00 & 0.59 \\
\hline $22: 1 n-11$ & 1.58 & 1.61 & 1.31 & 1.42 & 1.25 & 1.41 & 0.04 & $1.59^{a}$ & $1.36^{b}$ & $1.33^{b}$ & 1.38 & 1.48 & 0.01 & 0.14 & 0.64 \\
\hline $22: 1 n-9$ & 0.36 & 0.37 & 0.33 & 0.35 & 0.30 & 0.33 & 0.01 & $0.37^{a}$ & $0.34^{a b}$ & $0.32^{b}$ & 0.33 & 0.35 & 0.04 & 0.24 & 0.83 \\
\hline $22: 4 n-6$ & 0.08 & 0.06 & 0.09 & 0.06 & 0.08 & 0.08 & 0.00 & 0.07 & 0.08 & 0.08 & $0.09^{A}$ & $0.07^{\mathrm{B}}$ & 0.28 & 0.00 & 0.11 \\
\hline $22: 5 n-6$ & 0.29 & 0.22 & 0.36 & 0.23 & 0.35 & 0.31 & 0.01 & $0.26^{b}$ & $0.30^{a}$ & $0.33^{a}$ & $0.33^{A}$ & $0.25^{\mathrm{B}}$ & 0.00 & 0.00 & 0.02 \\
\hline $22: 5 n-3$ & 1.41 & 0.94 & 1.58 & 1.03 & 1.50 & 1.28 & 0.06 & 1.18 & 1.31 & 1.39 & $1.50^{\mathrm{A}}$ & $1.08^{\mathrm{B}}$ & 0.07 & 0.00 & 0.15 \\
\hline $22: 6 n-3$ & 16.0 & 12.1 & 18.7 & 12.4 & 16.9 & 15.6 & 0.68 & 14.0 & 15.6 & 16.3 & $17.2^{\mathrm{A}}$ & $13.4^{\mathrm{B}}$ & 0.15 & 0.00 & 0.11 \\
\hline Total SFA & 21.0 & 19.7 & 22.5 & 20.5 & 26.6 & 20.7 & 0.61 & $20.3^{\mathrm{b}}$ & $21.5^{\mathrm{b}}$ & $23.6^{a}$ & $23.4^{\mathrm{A}}$ & $20.3^{B}$ & 0.00 & 0.00 & 0.01 \\
\hline Total MUFA & 39.4 & 45.8 & 26.5 & 44.9 & 36.1 & 41.8 & 0.97 & $42.6^{a}$ & $40.7^{a b}$ & $39.0^{b}$ & $37.3^{\mathrm{B}}$ & $44.2^{\mathrm{A}}$ & 0.01 & 0.00 & 0.35 \\
\hline Total n-6 & 12.9 & 13.5 & 10.9 & 12.6 & 9.1 & 11.5 & 0.35 & $13.2^{\mathrm{a}}$ & $11.7^{\mathrm{b}}$ & $10.3^{c}$ & $11.0^{\mathrm{B}}$ & $12.5^{\mathrm{A}}$ & 0.00 & 0.00 & 0.00 \\
\hline Total n-6 LC-PUFA & 1.91 & 1.56 & 2.04 & 1.62 & 1.95 & 1.79 & 0.04 & $1.73^{b}$ & $1.83^{\mathrm{a}}$ & $1.86^{\mathrm{a}}$ & $1.96^{\mathrm{A}}$ & $1.66^{\mathrm{B}}$ & 0.00 & 0.00 & 0.00 \\
\hline Total n-3 & 25.7 & 20.1 & 29.0 & 21.1 & 26.9 & 25.0 & 0.90 & 22.9 & 25.0 & 25.9 & $27.2^{\mathrm{A}}$ & $22.1^{\mathrm{B}}$ & 0.14 & 0.00 & 0.15 \\
\hline Total n-3 LC-PUFA & 22.3 & 16.3 & 26.0 & 17.3 & 24.3 & 21.4 & 0.96 & 19.3 & 21.7 & 22.8 & $24.2^{\mathrm{A}}$ & $18.4^{\mathrm{B}}$ & 0.06 & 0.00 & 0.15 \\
\hline $\mathrm{EPA}+\mathrm{DHA}$ & 20.2 & 14.8 & 23.7 & 15.6 & 22.2 & 19.5 & 0.90 & 17.5 & 19.7 & 20.8 & $22.0^{\mathrm{A}}$ & $16.6^{\mathrm{B}}$ & 0.06 & 0.00 & 0.15 \\
\hline EPA/ARA & 6.31 & 5.50 & 6.21 & 5.82 & 6.07 & 5.90 & 0.10 & 5.91 & 6.02 & 5.99 & $6.20^{\mathrm{A}}$ & $5.74^{\mathrm{B}}$ & 0.87 & 0.02 & 0.33 \\
\hline DHAVEPA & 3.77 & 4.44 & 3.73 & 3.87 & 3.23 & 4.05 & 0.10 & $4.11^{\mathrm{a}}$ & $3.80^{\mathrm{ab}}$ & $3.64^{b}$ & $3.58^{\mathrm{B}}$ & $4.12^{\mathrm{A}}$ & 0.01 & 0.00 & 0.03 \\
\hline$n-6 / n-3$ & 0.51 & 0.69 & 0.38 & 0.60 & 0.34 & 0.46 & 0.03 & $0.60^{a}$ & $0.49^{a b}$ & $0.40^{b}$ & $0.41^{\mathrm{B}}$ & $0.59^{A}$ & 0.00 & 0.00 & 0.54 \\
\hline
\end{tabular}

Values are mean of triplicate groups $(n=3)$. Different superscript letters indicate significant difference among treatments $(P<0.05)$. 
TABLE 7 | Serum biochemical parameters at $24 \mathrm{~h}$ post-feeding in European grayling (Thymallus thymallus).

\begin{tabular}{|c|c|c|c|c|c|c|c|c|c|c|}
\hline Diets & Protein (\%) & Lipid (\%) & TP & TG & $\mathrm{CHO}$ & GLU & ALT & AST & ALP & LDH \\
\hline \multicolumn{11}{|c|}{ Individual treatment means } \\
\hline P30L10 & 30 & 10 & 4.08 & 264 & 218 & 76.4 & 48.3 & 835 & 380 & 7,704 \\
\hline P40L10 & 40 & 10 & 4.38 & 256 & 242 & 75.6 & 32.2 & 719 & 344 & 5,192 \\
\hline P50L10 & 50 & 10 & 4.51 & 220 & 277 & 83.1 & 33.0 & 611 & 363 & 5,767 \\
\hline P30L20 & 30 & 20 & 4.48 & 473 & 279 & 103 & 28.3 & 592 & 386 & 4,437 \\
\hline P40L20 & 40 & 20 & 4.16 & 426 & 258 & 84.4 & 28.7 & 519 & 332 & 4,389 \\
\hline P50L20 & 50 & 20 & 4.49 & 379 & 277 & 96.2 & 34.3 & 605 & 350 & 3,416 \\
\hline SEM & & & 0.07 & 23.2 & 6.11 & 2.73 & 2.16 & 33.4 & 8.02 & 330 \\
\hline \multicolumn{11}{|c|}{ Means of main effects } \\
\hline & 30 & & 4.28 & $368^{a}$ & $248^{b}$ & $89.8^{a}$ & 38.3 & 713 & 383 & $6,070^{a}$ \\
\hline & 40 & & 4.27 & $341^{a}$ & $250^{b}$ & $80.0^{b}$ & 30.4 & 619 & 338 & $4,790^{b}$ \\
\hline & 50 & & 4.50 & $300^{b}$ & $277^{a}$ & $89.7^{a}$ & 33.7 & 608 & 357 & $4,591^{b}$ \\
\hline & & 10 & 4.32 & $247^{\mathrm{B}}$ & $246^{\mathrm{B}}$ & $78.4^{\mathrm{B}}$ & $37.9^{A}$ & $721^{A}$ & 363 & $6,221^{A}$ \\
\hline & & 20 & 4.37 & $426^{A}$ & $271^{A}$ & $94.6^{A}$ & $30.4^{\mathrm{B}}$ & $572^{\mathrm{B}}$ & 356 & $4,080^{B}$ \\
\hline \multicolumn{11}{|c|}{ Two-way ANOVA (P-value) } \\
\hline Protein & & & 0.27 & 0.00 & 0.01 & 0.03 & 0.20 & 0.25 & 0.09 & 0.00 \\
\hline Lipid & & & 0.70 & 0.00 & 0.00 & 0.00 & 0.04 & 0.02 & 0.68 & 0.00 \\
\hline Interaction & & & 0.17 & 0.11 & 0.01 & 0.07 & 0.06 & 0.20 & 0.85 & 0.00 \\
\hline
\end{tabular}

Values are mean of triplicate groups $(n=18)$. Different superscript letters indicate significant difference among treatments $(P<0.05)$. TP, Total protein $\left(g d l^{-1}\right)$ : TG,

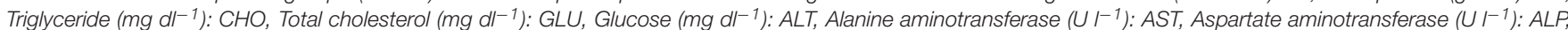
Alkaline phosphatase $\left(U I^{-1}\right)$ : $L D H$, Lactate dehydrogenase $\left(\mathrm{UI}^{-1}\right)$.

TABLE 8 | Serum antioxidant and innate immune parameters at $24 \mathrm{~h}$ post-feeding in European grayling (Thymallus thymallus).

\begin{tabular}{|c|c|c|c|c|c|c|c|c|}
\hline Diets & Protein (\%) & Lipid (\%) & SOD & CAT & MDA & LYZ & MPO & AP \\
\hline & & & \multicolumn{6}{|c|}{ Individual treatment means } \\
\hline P30L10 & 30 & 10 & 49.1 & 36.4 & 170 & 12.7 & 1.33 & 30.2 \\
\hline P40L10 & 40 & 10 & 57.7 & 34.2 & 213 & 12.2 & 1.45 & 35.8 \\
\hline P50L10 & 50 & 10 & 62.4 & 36.8 & 243 & 12.9 & 1.47 & 33.6 \\
\hline P30L20 & 30 & 20 & 55.6 & 38.5 & 254 & 12.7 & 1.75 & 33.9 \\
\hline P40L20 & 40 & 20 & 58.3 & 37.2 & 227 & 12.4 & 1.70 & 34.4 \\
\hline P50L20 & 50 & 20 & 49.2 & 36.4 & 274 & 12.4 & 1.53 & 33.2 \\
\hline \multirow[t]{7}{*}{ SEM } & & & 1.58 & 0.61 & 9.96 & 0.45 & 0.04 & 0.62 \\
\hline & & & \multicolumn{6}{|c|}{ Means of main effects } \\
\hline & 30 & & 52.3 & 37.4 & $212^{b}$ & 12.7 & 1.54 & 32.0 \\
\hline & 40 & & 58.0 & 35.7 & $220^{a b}$ & 12.3 & 1.57 & 35.1 \\
\hline & 50 & & 55.8 & 36.6 & $258^{a}$ & 12.6 & 1.50 & 33.4 \\
\hline & & 10 & 56.4 & 35.8 & $208^{B}$ & 12.6 & $1.42^{\mathrm{B}}$ & 33.2 \\
\hline & & 20 & 54.4 & 37.3 & $252^{\mathrm{A}}$ & 12.5 & $1.66^{\mathrm{A}}$ & 33.8 \\
\hline \multicolumn{9}{|c|}{ Two-way ANOVA (P-value) } \\
\hline Protein & & & 0.22 & 0.53 & 0.04 & 0.95 & 0.63 & 0.11 \\
\hline Lipid & & & 0.43 & 0.23 & 0.01 & 0.94 & 0.00 & 0.59 \\
\hline Interaction & & & 0.02 & 0.53 & 0.14 & 0.95 & 0.08 & 0.16 \\
\hline
\end{tabular}

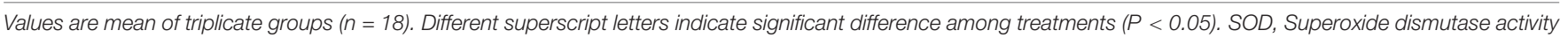

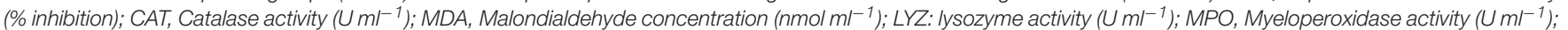
$A P$, Antiprotease activity (\% trypsin inhibition).

ranging from 22.4 to $33.02 \mathrm{~g} \mathrm{MJ}^{-1}$ on growth of Manchurian trout (Brachymystax lenok). Their findings showed that although the best growth rate occurs at $50 \%$ protein and $8 \%$ lipid with $\mathrm{P} / \mathrm{E}$ ratio of $29.36 \mathrm{~g} \mathrm{MJ}^{-1}$, similar WG and SGR could be achieved at $45 \%$ protein and $16 \%$ lipid levels with $\mathrm{P} / \mathrm{E}$ ratio of $23.68 \mathrm{~g}$ $\mathrm{MJ}^{-1}$. The results of the present study revealed that growth rate of grayling was influenced by protein content of feed and that $40 \%$ protein produces a comparable growth rate to the group fed the diet with $50 \%$ protein. Moreover, in this study the highest growth rate $(266 \% \mathrm{WG})$ was obtained at $50 \%$ protein and $20 \%$ lipid levels with $\mathrm{P} / \mathrm{E}$ ratio of $22.1 \mathrm{~g} \mathrm{MJ}^{-1}$ which was comparable to the growth rate of the group received the diet 
TABLE 9 | Blood plasma ammonia $\left(\mathrm{NH}_{3}-\mu \mathrm{mol} \mathrm{I}^{-1}\right)$ concentration at $5 \mathrm{~h}$ post-feeding, and rearing water ammonium nitrogen $\left(\mathrm{NH}_{4}{ }^{+}-\mathrm{N}-\mathrm{mg} \mathrm{kg}^{-1} \mathrm{~h}^{-1}\right)$ concentration at 5 and $24 \mathrm{~h}$ post-feeding in European grayling (Thymallus thymallus).

\begin{tabular}{|c|c|c|c|c|c|}
\hline \multirow[t]{2}{*}{ Diets } & \multirow[t]{2}{*}{ Protein (\%) } & \multirow[t]{2}{*}{ Lipid (\%) } & \multirow{2}{*}{$\frac{\text { Plasma }}{5 \mathrm{~h}}$} & \multicolumn{2}{|c|}{ Rearing water } \\
\hline & & & & $5 \mathrm{~h}$ & $24 \mathrm{~h}$ \\
\hline & & & \multicolumn{3}{|c|}{ Individual treatment means } \\
\hline P30L10 & 30 & 10 & 630 & 11.1 & 14.0 \\
\hline P40L10 & 40 & 10 & 595 & 13.5 & 14.9 \\
\hline P50L10 & 50 & 10 & 648 & 17.0 & 20.8 \\
\hline P30L20 & 30 & 20 & 503 & 9.80 & 10.9 \\
\hline P40L20 & 40 & 20 & 556 & 8.90 & 11.6 \\
\hline P50L20 & 50 & 20 & 625 & 11.5 & 15.3 \\
\hline \multirow[t]{7}{*}{ SEM } & & & 20.2 & 0.72 & 0.91 \\
\hline & & & \multicolumn{3}{|c|}{ Means of main effects } \\
\hline & 30 & & 566 & $10.5^{b}$ & $12.4^{\mathrm{b}}$ \\
\hline & 40 & & 576 & $11.2^{b}$ & $13.3^{b}$ \\
\hline & 50 & & 637 & $14.3^{\mathrm{a}}$ & $18.1^{a}$ \\
\hline & & 10 & 624 & $13.9^{\mathrm{A}}$ & $16.6^{A}$ \\
\hline & & 20 & 562 & $10.1^{\mathrm{B}}$ & $12.6^{\mathrm{B}}$ \\
\hline \multicolumn{6}{|c|}{ Two-way ANOVA (P-value) } \\
\hline Protein & & & 0.31 & 0.01 & 0.00 \\
\hline Lipid & & & 0.13 & 0.00 & 0.00 \\
\hline Interaction & & & 0.52 & 0.12 & 0.65 \\
\hline
\end{tabular}

Values are mean of triplicate groups $(n=3)$. Different superscript letters indicate significant difference among treatments $(P<0.05)$.

containing $40 \%$ protein and $20 \%$ lipid (257\% WG) with $\mathrm{P} / \mathrm{E}$ ratio of $18 \mathrm{~g} \mathrm{MJ}^{-1}$. Similarly, Azevedo et al. (2002) found no significant improvement in growth performance of Atlantic salmon (Salmo salar) when $\mathrm{P} / \mathrm{E}$ ratio increased from 18 to $20 \mathrm{~g} \mathrm{MJ}^{-1}$. Also, Green and Hardy (2008) found similar growth performance for rainbow trout (Oncorhynchus mykiss) fed diets with $\mathrm{P} / \mathrm{E}$ ratios of 18, 22, and $24 \mathrm{~g} \mathrm{MJ}^{-1}$.

Fish FI decreased at higher protein and lipid levels which agrees with studies on bagrid catfish (Pseudobagrus fulvidraco) (Kim and Lee, 2005), brown-marbled grouper (Epinephelus fuscoguttatus) (Shapawi et al., 2014) and hybrid grouper (Epinephelus $\times$ E. lanceolatus) (Rahimnejad et al., 2015). This could be due to increased feed energy content as lesser feed would be consumed by fish to meet its energy requirement (Kaushik and Medale, 1994). Moreover, when fish are offered diets with a protein content below the requirement level, they would consume more feed to gain sufficient protein needed for supporting growth and metabolism while at optimum or higher dietary protein levels lesser diet would be needed. Our results revealed the enhancement of $\mathrm{FE}$ as protein and lipid levels increased which is consistent with studies on masu salmon (Lee and Kim, 2001), Manchurian trout (Xu et al., 2015), brown trout (Salmo trutta fario) (Wang C. et al., 2018), Japanese seabass (Lateolabrax japonicus) (Ai et al., 2004) and black seabass (Centropristis striata) (Alam et al., 2009). Xu et al. (2015) showed that enhancing fat content of feed from 8 to $16 \%$ enhances PER in Manchurian trout at dietary protein levels of $40-45 \%$. Moreover, Lee and Kim (2001) showed the significant improvement of PER in masu salmon by increasing energy content of diet from 19 to $21 \mathrm{MJ} \mathrm{kg}^{-1}$ at protein levels of 30,40 , and $50 \%$. Similarly, in this study PER was improved by increasing dietary lipid level from 10 to $20 \%$ corresponding to $2 \mathrm{MJ} \mathrm{kg}^{-1}$ increase in dietary energy content. Improvement of PER at higher dietary fat level has also been shown in pikeperch (Sander lucioperca) (Schulz et al., 2008), Japanese seabass (Ai et al., 2004), and yellow drum (Nibea albiflora) (Wang L. et al., 2018). On the other hand, in this study PER showed a decreasing tendency by increasing dietary protein level which is parallel to earlier findings in pikeperch (Schulz et al., 2008), red-spotted grouper (Epinephelus akaara) (Wang et al., 2017) and hybrid grouper (Jiang et al., 2015; Rahimnejad et al., 2015). The underlying reason for the higher PER at lower dietary protein level could be the efficient utilization of protein at low protein levels (Catacutan et al., 2001). However, no protein sparing effect of lipid could be found in this study as dietary lipid level did not influence fish growth performance. This is consistent with studies on sunshine bass (Morone chrysops × M. saxatilis) (Gallagher, 1999), redspotted grouper (Wang et al., 2017), hybrid grouper (Jiang et al., 2015) and hybrid snakehead (Channa maculata $\times$ C. argus) (Zhang et al., 2017). These findings may signify the lower ability of grayling juveniles in oxidizing lipids and using them as an energy source. This was confirmed by increased VSI and IPF at increased lipid level indicating the lipid accumulation in fish abdominal cavity rather than being oxidized for energy production. Moreover, an interaction of dietary protein and lipid levels was found on $\mathrm{K}$ where increasing protein level at $10 \%$ lipid led to numerical reduction of $\mathrm{K}$ while an opposite trend was found at $20 \%$ lipid. This could be due to lipid accumulation in abdominal cavity at the higher dietary lipid level resulting in the alteration of fish weight/length ratio. 
Whole-body lipid content was impacted by interaction of protein and lipid and moisture content decreased by increasing fat content of diet. Moreover, muscle lipid concentration reflected the dietary fat content. Likewise, Lee and Kim (2001) showed the reduction of whole-body moisture and increment of lipid content in masu salmon at increased dietary fat. Also, a rainbow trout study showed the enhancement of whole-body lipid and reduction of moisture content at increased lipid level (Yamamoto et al., 2000). Similar observations have been reported in Manchurian trout (Xu et al., 2015), giant croaker (Nibea japonica) (Chai et al., 2013) and Channa striata (Hua et al., 2019). Ash content of muscle increased by increasing protein level and reflected the dietary ash content.

Dietary lipid level is one of the primary factors that influences the muscle fatty acid composition (Lopez et al., 2009). Increasing fat content of feed in this study resulted in reduced muscle ARA, EPA, DHA, total n-6 LC-PUFA, total n-3, total n-3 LC-PUFA, and EPA/ARA ratio whereas an increasing trend was observed for LA, LNA, total MUFA, total $n-6$, and ratios of DHA/EPA and n-6/n-3. Similarly, Kim et al. (2012) reported that higher fat content in the diet resulted in reduced EPA, DHA, n-3 PUFA and n-3 HUFA in far eastern catfish whole-body. A hybrid grouper study revealed only increased muscle LNA content at increased dietary fat level (Rahimnejad et al., 2015) which is consistent with the current study. Moreover, Arslan et al. (2013) found the enhancement of whole-body LA, LNA, EPA, DHA, total PUFA, total n-3 and total n- 6 contents and reduction of ARA content by increasing lipid level in diets for South American catfish (Pseudoplatystoma sp.). The increment of muscle LA, LNA, total MUFA and total n- 6 contents and n-6/n-3 ratio at higher dietary fat level could be ascribed to increased inclusion level of soybean oil. Also, the reduction of ARA, EPA and DHA concentrations in response to increased dietary fat content may indicated the limited bioconversion of these fatty acids from LA and LNA (Arslan et al., 2013). Thomassen et al. (2017) showed that long term feeding of a diet deficient in $\mathrm{n}-3$ fatty acids is associated with significantly increased proportion of 20:3n-9 (Mead acid) in different organs and muscle of Atlantic salmon. The low level of Mead acid in muscle of grayling, irrespective of dietary groups, indicates the lack of essential FAs deficiency.

Blood biochemical parameters and enzymes activity are used as key means of surveying the fish health and nutritional status ( $\mathrm{Li}$ et al., 2015). Lipoproteins are the most common transported form of lipids in fish (Wang et al., 2017). Plasma/serum lipoprotein, TG and CHO concentrations could be used as useful indicators for evaluation of the endogenous lipid transport (Du et al., 2005; Chen et al., 2012). Dietary lipid and protein contents influence serum/plasma CHO concentration (NRC, 2011; Jiang et al., 2015). Increment of serum $\mathrm{CHO}$ concentration in fish fed high fat diets confirmed the earlier findings in hybrid grouper (Jiang et al., 2015), red-spotted grouper (Wang et al., 2017) and hybrid snakehead (Channa maculata $\times$ C. argus) (Zhang et al., 2017). Additionally, we detected elevated serum $\mathrm{CHO}$ concentration at higher dietary protein levels which agrees with the study on red-spotted grouper (Wang et al., 2017). Several authors noted that TG level in blood has an inverse relationship with protein content of feed (Zhang et al., 2010; Jin et al., 2015) but has a positive relationship with fat content ( $\mathrm{Li}$ et al., 2017; Cao et al., 2019). Likewise, in this study serum TG concentration doubled at higher dietary lipid level indicating more active transport of lipids. Whilst, increasing dietary protein level led to significant reduction of TG concentration which is consistent with findings in hybrid grouper (Jiang et al., 2015). There are several reports indicating that increased blood TG concentration in fish might be used as an indicator of poor health (Kikuchi et al., 2009). Wang et al. (2019) reported the reduction of serum TG concentration in black sea bream (Acanthopagrus schlegelii) by increasing dietary $\mathrm{P} / \mathrm{E}$ ratio, and claimed that higher serum TG concentration at low $\mathrm{P} / \mathrm{E}$ ratios could be a symptom of declined health status as it was coupled with lower growth rate and antioxidant capacity. The increment of serum GLU concentration at higher lipid level in this study agrees with the researches on common carp (Cyprinus carpio) (Aminikhoei et al., 2015), rohu (Labeo rohita) (Kumar et al., 2019), and largemouth bass (Li et al., 2020). The increment of GLU concentration in serum of fish fed high fat diet could be associated with impairment of GLU homeostasis as documented in rainbow trout (Figueiredo-Silva et al., 2012). Also, Panserat et al. (2002) reported ineffective control of glycemia in rainbow trout fed a high fat diet in comparison to those received a low fat diet. Furthermore, these authors found the significant enhancement of hepatic glucose-6-phosphate (an enzyme involved in glucogenesis) mRNA expression and enzyme activity in trout fed high fat diet. ALT and AST are considered as non-functional enzymes found in cells of liver, kidney, and other organs. Their elevated levels in blood stream is used as an indicator of liver abnormal function (Bain, 2003). Moreover, the surge in their serum/plasma concentration could also occur in response to various stressors such as ammonia and nitrite toxicity (Das et al., 2004; Choi et al., 2007). The results exhibited the remarkable reduction of serum ALT and AST activities by increasing dietary fat content. Similarly, Zhang et al. (2018a) reported decreased serum ALT and AST activities in barbless carp (Cyprinus pellegrini) at increased dietary fat level. This could be because of reduced catabolism of protein for energy production at higher dietary lipid level leading to reduced ammonia production. This was further confirmed by decreased plasma ammonia concentration and ammonia excretion to the rearing water at higher dietary lipid level in this study. Amino acids oxidation demands excessive energy for deamination and excretion and causes stress in fish (Walton, 1985). Moreover, increasing dietary protein and lipid levels led to decreased serum LDH activity which is also considered as a marker of stress response in fish (Chatterjee et al., 2006; Kumar et al., 2018). Likewise, Li et al. (2020) showed that increasing dietary protein level results in reduced serum and liver LDH activity in largemouth bass (Micropterus salmoides). Also, Hemre and Sandnes (1999) showed the remarkable reduction of plasma LDH activity in Atlantic salmon by increasing dietary lipid level.

Dietary $\mathrm{P} / \mathrm{E}$ ratio may influence the antioxidant system by providing sufficient supply of indispensable amino acids and fatty acids (Wang et al., 2019). A yellow catfish (Pelteobagrus fulvidraco) study showed that optimum dietary protein/lipid ratio improves antioxidant capacity through promoting the immune cells proliferation by providing sufficient protein 
(Zhang et al., 2018b). However, the effect of dietary treatments was null on serum SOD and CAT activities in this research similar to a black sea bream study (Wang et al., 2019). MDA is an end-product of lipid peroxidation and its elevated level in serum is used as a valuable marker of oxidative damage. Our results indicated the enhancement of serum MDA concentration by increasing protein and lipid levels. Likewise, increased serum/plasma/liver MDA concentration at higher lipid levels has been reported in black sea bream (Wang et al., 2019), triploid rainbow trout (Meng et al., 2019) and largemouth bass (Yin et al., 2021). Żebrowska et al. (2019) showed that high protein diet leads to increased plasma MDA concentration in rats. These authors suggested that the ingestion of high protein diet may result in redox imbalance in healthy non-obese rats. It has been well established that immune response in fish is tightly linked to nutritional status (Lygren and Waagbo, 1999). Concentration and type of lipids are among the nutritional factors that modulate different components of the immune system in animals (Korver et al., 1995; Lin and Shiau, 2003). A Malabar grouper (Epinephelus malabaricus) study revealed the promotion of lysozyme and ACH50 activities by enhancing fat content of feed (Lin and Shiau, 2003). Similarly, our results revealed the enhancement of serum MPO activity by increasing dietary lipid concentration, however, lysozyme and antiprotease activities remained unaffected. Lipids, their constituent FAs, and compounds derived from metabolism of FAs such as eicosanoids play key roles in immune function of finfish species (Higgs and Dong, 2000). Dietary lipid influences a wide range of immune parameters including proliferation of lymphocytes, synthesis of cytokines, natural killer cell activity, and phagocytosis. It is also speculated that FAs may modulate immune system through modifying membrane fluidity, formation of lipid peroxide, synthesis of eicosanoids, and regulating the expression of associated genes (De Pablo Martinez and Álvarez De Cienfuegos, 2000). Moreover, there are several reports indicating that $\mathrm{DHA} / \mathrm{EPA}$ ratio impacts immune response in fish (Montero et al., 2004; Zuo et al., 2012). For instance, Zhang et al. (2019) showed that DHA/EPA ratio of 1.48 significantly enhances serum immune indices in juvenile golden pompano (Trachinotus ovatus).

Ammonia production could be used as a useful indicator of protein metabolism in fish where protein retention is inversely correlated with produced ammonia (Webb and Gatlin, 2003). Inadequate supply of non-protein energy sources in the diet could result in catabolism of part of protein to meet the energy requirement of fish for maintenance and growth which is accompanied with increased ammonia production and excretion to the rearing water (Hung et al., 2017). Likewise, in this study increasing lipid level from 10 to $20 \%$ led to remarkable reduction of water ammonium concentration at 5 and $24 \mathrm{~h}$ post feeding indicating that increasing lipid content in diets for grayling can perhaps improve dietary protein utilization and reduce ammonia excretion. Furthermore, at higher protein levels more protein is catabolized leading to higher ammonia excretion (Kim et al., 1991; Yang et al., 2002). In the current study, water ammonium concentration increased at both samplings when dietary protein level increased from 40 to $50 \%$. Similarly, Yang et al. (2002) reported the enhancement of accumulated ammonia concentration in silver perch (Bidyanus bidyanus) by increasing dietary protein level.

\section{CONCLUSION}

These results suggest that $40 \%$ protein can support growth of European grayling with average body weight of 25-60 g, and increasing dietary fat content to $20 \%$ can enhance FE and PER. Increasing dietary protein level led to increased muscle ARA, EPA and total n-6 LC-PUFA contents whereas the elevation of dietary lipid level resulted in decreased EPA, DHA, and total n-3 LC-PUFA and n-6 LC-PUFA. In addition, decreased DHA/EPA and $n-6 / n-3$ ratios were observed as dietary protein content increased while these ratios increased at higher dietary lipid level. The reduction of serum ALT and AST activities indicated the improvement of liver health at the higher lipid level. Moreover, increasing dietary fat content resulted in decreased ammonia excretion to the rearing water.

\section{DATA AVAILABILITY STATEMENT}

The raw data supporting the conclusions of this article will be made available by the authors, without undue reservation.

\section{ETHICS STATEMENT}

All the experimental procedures were performed in compliance with valid legislative regulations in Czech Republic (law no. 166/1996 and no. 246/1992); the permit was issued to no. 2293/2015-MZE-17214 and no. 55187/2016-MZE17214. All samplings were carried out with the relevant permission from the Departmental Expert Committee for Authorization of Experimental Projects of the Ministry of Education, Youth and Sports of the Czech Republic (permit no. MSMT 4394/2017-2).

\section{AUTHOR CONTRIBUTIONS}

SR, KD, and TP: conceptualization, validation, visualization, writing, and review and editing. SR, KD, OM, and JK: methodology. SR: investigation and manuscript drafting. SR and MI: formal analysis and collection and interpretation of data. TP: funding acquisition, project administration, and resources. All authors contributed to the article and approved the submitted version.

\section{FUNDING}

This study was financially supported by the Ministry of Agriculture of the Czech Republic, project NAZV QK1920326, and by the Ministry of Education, Youth and Sports of the Czech Republic, project Biodiversity (CZ.02.1.01./0.0/0.0/16_025/0007370). 


\section{REFERENCES}

Ai, Q. H., Mai, K. S., Li, H. T., Zhang, C. X., Zhang, L., Duan, Q. Y., et al. (2004). Effects of dietary protein to energy ratios on growth and body composition of juvenile Japanese seabass, Lateolabrax japonicus. Aquaculture 230, 507-516. doi: 10.1016/j.aquaculture.2003.09.040

Alam, M. S., Watanabe, W. O., Carroll, P. M., and Rezek, T. (2009). Effects of dietary protein and lipid levels on growth performance and body composition of black sea bass Centropristis striata (Linnaeus 1758) during grow-out in a pilot-scale marine recirculating system. Aquac. Res. 40, 442-449. doi: 10.1111/j. 1365-2109.2008.02113.x

Ali, M. Z., and Jauncey, K. (2005). Approaches to optimizing dietary protein to energy ratio for African catfish Clarias gariepinus (Burchell, 1822). Aquac. Nutr. 11, 95-101. doi: 10.1111/j.1365-2095.2004.00325.x

Aminikhoei, Z., Choi, J., and Lee, S. M. (2015). Optimal dietary protein and lipid levels for growth of juvenile israeli carp Cyprinus carpio. Fish. Aquat. Sci. 18, 265-271. doi: 10.5657/FAS.2015.0265

Asociation of Official Analytical Chemists (AOAC). (2002). Official Methods of Analysis of Official Analytical Chemists International, 17th Edn. Arlington, VA: Association of Official Analytical Chemists.

Arslan, M., Dabrowski, K., Ferrer, S., Dietrich, M., and Rodriguez, G. (2013). Growth, body chemical composition and trypsin activity of South American catfish, surubim (Pseudoplatystoma sp.) juveniles fed different dietary protein and lipid levels. Aquac. Res. 44, 760-771. doi: 10.1111/j.1365-2109.2011.03081.

Azevedo, P. A., Bureau, D. P., Leeson, S., and Cho, Y. (2002). Growth and efficiency of feed usage by Atlantic salmon (Salmo salar) fed diets with different dietary protein: energy ratios at two feeding levels. Fish. Sci. 68, 878-888. doi: 10.1046/ j.1444-2906.2002.00506.x

Bain, P. (2003). "Liver," in Duncan \& Prasse's Veterinary Laboratory Medicine: Clinical Pathology, eds K. Latimer, K. S. Mahafey, and E. A. Prasse (Ames, IA: Iowa State Press), 193-214.

Bolotov, I. N., Novoselov, A. P., Bespalaya, Y. V., and Usacheva, O. V. (2012). Feeding of European grayling Thymallus thymallus (Salmoniformes: Thymallidae) in the early winter period in the pymvashor stream (Subarctic hydrothermal system). J. Ichthyol. 52, 180-184. doi: 10.1134/S0032945212020038

Cao, X.-F., Liu, W.-B., Zheng, X.-C., Yuan, X.-Y., Wang, C.-C., and Jiang, G.-Z. (2019). Effects of high-fat diets on growth performance, endoplasmic reticulum stress and mitochondrial damage in blunt snout bream Megalobrama amblycephala. Aquac. Nutr. 25, 97-109. doi: 10.1111/anu.12834

Carlstein, M. (2004). Growth and survival of European grayling reared at different stocking densities. Aquac. Int. 3, 260-264.

Catacutan, M. R., Pagador, G. E., and Teshima, S. (2001). Effect of dietary protein and lipid levels and protein to energy ratios on growth, survival and body composition of the mangrove red snapper, Lutjanus argentimaculatus (Forsskal 1775). Aquac. Res. 32, 811-818. doi: 10.1046/j.1365-2109.2001.00618.x

Chai, X. J., Ji, W. X., Han, H., Dai, Y. X., and Wang, Y. (2013). Growth, feed utilization, body composition and swimming performance of giant croaker, Nibea japonica Temminck and Schlegel, fed at different dietary protein and lipid levels. Aquac. Nutr. 19, 928-935. doi: 10.1111/anu.12038

Chatterjee, N., Pal, A. K., Das, T., Mohammed, M. S., Sarma, K., Venkateshwarlu, G., et al. (2006). Secondary stress responses in Indian major carps Labeo rohita (Hamilton), Catla catla (Hamilton) and Cirrhinus mrigala (Hamilton) fry to increasing packing densities. Aquac. Res. 37, 472-476. doi: 10.1111/j.1365-2109. 2006.01469.x

Chen, Y.-J., Tian, L.-X., Yang, H.-J., Chen, P.-F., Yuan, Y., Liu, Y.-J., et al. (2012). Effect of protein and starch level in practical extruded diets on growth, feed utilization, body composition, and hepatic transaminases of juvenile grass Carp, Ctenopharyngodon idella. J. World Aquac. Soc. 43, 187-197. doi: 10.1111/j.17497345.2012.00549.x

Christie, W. W. (1989). Gas Chromatography and Lipids. Glasgow: The Oily Press.

Choi, C. Y., Min, B. H., Jo, P. G., and Chang, Y. J. (2007). Molecular cloning of PEPCK and stress response of black porgy (Acanthopagrus schlegeli) to increased temperature in freshwater and seawater. Gen. Comp. Endocrinol. 152, 47-53. doi: 10.1016/j.ygcen.2007.02.019

Das, P. C., Ayappan, A. J., Jena, J. K., and Das, B. K. (2004). Acute toxicity of ammonia and its sub-lethal effects on selected haematological and enzymatic parameters of mrigal, Cirrhinus mrigala (Hamilton). Aquac. Res. 35, 134-143. doi: 10.1111/j.1365-2109.2004.00994.x

De Pablo Martinez, M. A., and Álvarez De Cienfuegos, G. (2000). Modulatory effects of dietary lipids on immune system functions. Immunol. Cell Biol. 78, 31-39. doi: 10.1046/j.1440-1711.2000.00875.x

Du, Z. Y., Liu, Y. J., Tian, L. X., Wang, J. T., Wang, Y., and Liang, G. Y. (2005). Effect of dietary lipid level on growth, feed utilization and body composition by juvenile grass carp (Ctenopharyngodon idella). Aquac. Nutr. 11, 139-146. doi: 10.1111/j.1365-2095.2004.00333.x

Ellis, A. E., Stolen, J. S., Fletcher, T. C., Anderson, D. P., Roberson, W. B., and Van muiswinker, W. B. (1990). Serum Antiprotease in Fish. Techniques in Fish Immunology. Fair Haven, NJ: SOS Publication.

Figueiredo-Silva, A. C., Panserat, S., Kaushik, S., Geurden, I., and Polakof, S. (2012). High levels of dietary fat impair glucose homeostasis in rainbow trout. J. Exp. Biol. 215, 169-178. doi: 10.1242/jeb.063933

Folch, J., Lees, M., and Stanley, G. H. S. (1957). A simple method for the isolation and purification of total lipides from animal tissues. J. Biol. Chem. 226, 497-509.

Gallagher, M. L. (1999). Growth responses, tissue composition, and liver enzyme changes in juvenile sunshine bass, Morone chrysops $\times$ M. saxatilis, associated with dietary protein and lipid level. J. Appl. Aquac. 9, 41-51. doi: 10.1300/ J028v09n04_05

Green, J. A., and Hardy, R. W. (2008). The effects of dietary protein:energy ratio and amino acid pattern on nitrogen utilization and excretion of rainbow trout Oncorhynchus mykiss (Walbaum). J. Fish Biol. 73, 663-682. doi: 10.1111/j.10958649.2008.01965.x

Halver, J. E., and Hardy, R. (2002). Fish Nutrition, 3rd Edn. San Diego, CA: Academic Press.

Hemre, G. I., and Sandnes, K. (1999). Effect of dietary lipid level on muscle composition in Atlantic salmon Salmo salar. Aquac. Nutr. 5, 9-16. doi: 10.1046/ j.1365-2095.1999.00081.x

Higgs, D. A., and Dong, F. M. (2000). "Lipids and fatty acids," in The Encyclopedia of Aquaculture, ed. R. R. Stickney (New York, NY: John Wiley and Sons, Inc), $1-20$.

Hua, K., Koppe, W., and Fontanillas, R. (2019). Effects of dietary protein and lipid levels on growth, body composition and nutrient utilization of Channa striata. Aquaculture 501, 368-373. doi: 10.1016/j.aquaculture.2018.11.054

Hung, L. T., Binh, V. T. T., Thanh Truc, N. T., Tham, L. H., and Ngoc Tran, T. (2017). Effects of dietary protein and lipid levels on growth, feed utilization and body composition in red-tailed catfish juveniles (Hemibagrus wyckioides, Chaux \& Fang 1949). Aquac. Nutr. 23, 367-374. doi: 10.1111/anu.12401

Izquierdo, M., Watanabe, T., Takeuchi, T., Arakawa, T., and Kitajima, C. (1990). "Optimum EFA levels in Artemia to meet the EFA requirements of red seabream (Pagrus major)," in The Current Status of Fish Nutrition in Aquaculture, eds M. Takeda and T. Watanabe (Tokyo: Tokyo University Fisheries), 221-232.

Jiang, S., Wu, X., Li, W., Wu, M., Luo, Y., Lu, S., et al. (2015). Effects of dietary protein and lipid levels on growth, feed utilization, body and plasma biochemical compositions of hybrid grouper (Epinephelus lanceolatus $\times$ Epinephelus fuscoguttatus) juveniles. Aquaculture 446, 148-155. doi: 10.1016/j. aquaculture.2015.04.034

Jin, Y., Tian, L., Xie, S., Guo, D., Yang, H., Liang, G., et al. (2015). Interactions between dietary protein levels, growth performance, feed utilization, gene expression and metabolic products in juvenile grass carp (Ctenopharyngodon idella). Aquaculture 437, 75-83. doi: 10.1016/j.aquaculture.2014.11.031

Kaushik, S. J., and Medale, F. (1994). Energy-requirements, utilization and dietary supply to salmonids. Aquaculture 124, 81-97. doi: 10.1016/0044-8486(94) 90364-6

Kikuchi, K., Furuta, T., Iwata, N., Onuki, K., and Noguchi, T. (2009). Effect of dietary lipid levels on the growth, feed utilization, body composition and blood characteristics of tiger puffer Takifugu rubripes. Aquaculture 298, 111-117. doi: 10.1016/j.aquaculture.2009.10.026

Kim, K. II, Kayesb, T. B., and Amundsonb, C. H. (1991). Purified diet development and re-evaluation of the dietary protein requirement of fingerling rainbow trout (Oncorhynchus mykiss). Aquaculture 96, 57-67.

Kim, K. D., Lim, S. G., Kang, Y. J., Kim, K. W., and Son, M. H. (2012). Effects of dietary protein and lipid levels on growth and body composition of juvenile far eastern catfish Silurus asotus. Asian Australas. J. Anim. Sci. 25, 369-374. doi: $10.5713 /$ ajas.2011.11089 
Kim, L. O., and Lee, S. M. (2005). Effects of the dietary protein and lipid levels on growth and body composition of bagrid catfish, Pseudobagrus fulvidraco. Aquaculture 243, 323-329. doi: 10.1016/j.aquaculture.2004.11.003

Korver, D. R., Wakenell, P., and Klasing, K. C. (1995). Dietary fish oil or lofrin, a 5-lipoxygenase inhibitor, decrease the growth- suppressing effects of coccidiosis in broiler chicks. Poult. Sci. 76, 1355-1363. doi: 10.1093/ps/76.10.1355

Koskinen, M. T., and Primmer, C. R. (2001). High throughput analysis of 17 microsatellite loci in grayling (Thymallus spp. Salmonidae). Conserv. Genet. 2, 173-177. doi: 10.1023/A:1011886412830

Kumar, S., Sahu, N. P., Deo, A. D., and Ranjan, A. (2019). Feeding de-oiled rice bran based diet with varying level of protein and lipid: effect on physiological responses of Labeo rohita. Aquaculture 498, 454-463. doi: 10. 1016/j.aquaculture.2018.08.068

Kumar, S., Sahu, N. P., Shamna, N., and Ranjan, A. (2018). Feeding higher level of de-oiled rice bran causes stress to Labeo rohita fingerlings. Aquaculture 484, 184-190. doi: 10.1016/j.aquaculture.2017.11.029

Lee, S. M., Jeon, I. G., and Lee, J. Y. (2002). Effects of digestible protein and lipid levels in practical diets on growth, protein utilization and body composition of juvenile rockfish (Sebastes schlegeli). Aquaculture 211, 227-239. doi: 10.1016/ S0044-8486(01)00880-8

Lee, S. M., and Kim, K. D. (2001). Effects of dietary protein and energy levels on the growth, protein utilization and body composition of juvenile masu salmon (Oncorhynchus masou Brevoort). Aquac. Res. 32, 39-45. doi: 10.1046/j.1355557x.2001.00004.x

Li, P.-Y., Wang, J.-Y., Song, Z.-D., Zhang, L.-M., Zhang, H., Li, X.-X., et al. (2015). Evaluation of soy protein concentrate as a substitute for fishmeal in diets for juvenile starry flounder (Platichthys stellatus). Aquaculture 448, 578-585. doi: 10.1016/j.aquaculture.2015.05.049

Li, W., Wen, X., Huang, Y., Zhao, J., Li, S., and Zhu, D. (2017). Effects of varying protein and lipid levels and protein-to-energy ratios on growth, feed utilization and body composition in juvenile Nibea diacanthus. Aquac. Nutr. 23, 1035-1047. doi: 10.1111/anu.12471

Li, X., Zheng, S., Ma, X., Cheng, K., and Wu, G. (2020). Effects of dietary protein and lipid levels on the growth performance, feed utilization, and liver histology of largemouth bass (Micropterus salmoides). Amino Acids 52, 1043-1061. doi: 10.1007/s00726-020-02874-9

Lin, Y. H., and Shiau, S. Y. (2003). Dietary lipid requirement of grouper, Epinephelus malabaricus, and effects on immune responses. Aquaculture 225, 243-250. doi: 10.1016/S0044-8486(03)00293-X

Lopez, L. M., Durazo, E., Teresa Viana, M., Drawbridge, M., and Bureau, D. P. (2009). Effect of dietary lipid levels on performance, body composition and fatty acid profile of juvenile white seabass, Atractoscion nobilis. Aquaculture 289, 101-105. doi: 10.1016/j.aquaculture.2009.01.003

Lygren, B., and Waagbo, R. (1999). Nutritional impacts on the chemiluminescent response of Atlantic salmon (Salmo salar L.) head kidney phagocytes, in vitro. Fish Shellfish Immunol. 9, 445-456. doi: 10.1006/fsim.1998.0203

Magnadóttir, B., Jonsdottir, H., Helgason, S., Bjornsson, B., Jørgensen, T., and Pilströ, L. (1999). Humoral immune parameters in Atlantic cod Gadus morhua L. I: the effects of environmental temperature. Comp. Biochem. Physiol. 122, 173-180. doi: 10.1016/s0305-0491(98)10156-6

Meng, Y., Qian, K., Ma, R., Liu, X., Han, B., Wu, J., et al. (2019). Effects of dietary lipid levels on sub-adult triploid rainbow trout (Oncorhynchus mykiss): 1. Growth performance, digestive ability, health status and expression of growthrelated genes. Aquaculture 513:734394. doi: 10.1016/j.aquaculture.2019.734394

Mir, I. N., Srivastava, P. P., Bhat, I. A., Jaffar, Y. D., Sushila, N., Sardar, P., et al. (2020). Optimal dietary lipid and protein level for growth and survival of catfish Clarias magur larvae. Aquaculture 520:734678. doi: 10.1016/j.aquaculture.2019. 734678

Montero, D., Socorro, J., Tort, L., Caballero, M. J., Robaina, L. E., Vergara, J. M., et al. (2004). Glomerulonephritis and immunosuppression associated with dietary essential fatty acid deficiency in gilthead sea bream, Sparus aurata L., juveniles. J. Fish Dis. 27, 297-306. doi: 10.1111/j.1365-2761.2004.00543.x

NRC (1993). Nutrient Requirements of Fish. Washington, DC: Natl Academy Press.

NRC (2011). Nutrient Requirements of Fish and Shrimp. Washington, DC: The National Academies Press.

Panserat, S., Perrin, A., and Kaushik, S. (2002). High dietary lipids induce liver glucose-6-phosphatase expression in rainbow trout (Oncorhynchus mykiss). J. Nutr. 132, 137-141. doi: 10.1093/jn/132.2.137
Quade, M. J., and Roth, J. A. (1997). A rapid, direct assay to measure degranulation of bovine neutrophil primary granules. Vet. Immunol. Immunopathol. 58, 239-248. doi: 10.1016/S0165-2427(97)00048-2

Rahimnejad, S., Bang, I. C., Park, J. Y., Sade, A., Choi, J., and Lee, S. M. (2015). Effects of dietary protein and lipid levels on growth performance, feed utilization and body composition of juvenile hybrid grouper, Epinephelus fuscoguttatus $\times$ E. lanceolatus. Aquaculture 446, 283-289. doi: 10.1016/j.aquacult ure.2015.05.019

Rahimnejad, S., and Lee, K. J. (2013). Dietary valine requirement of juvenile red sea bream Pagrus major. Aquaculture 416-417, 212-218. doi: 10.1016/j. aquaculture.2013.09.026

Rahimnejad, S., Lu, K., Wang, L., Song, K., Mai, K., Davis, D. A., et al. (2019). Replacement of fish meal with Bacillus pumillus SE5 and Pseudozyma aphidis ZR1 fermented soybean meal in diets for Japanese seabass (Lateolabrax japonicus). Fish Shellfish Immunol. 84, 987-997. doi: 10.1016/j.fsi.2018.11.009

Schulz, C., Huber, M., Ogunji, J., and Rennert, B. (2008). Effects of varying dietary protein to lipid ratios on growth performance and body composition of juvenile pike perch (Sander lucioperca). Aquac. Nutr. 14, 166-173. doi: 10.1111/j.13652095.2007.00516.x

Shapawi, R., Ebi, I., Yong, A. S. K., and Ng, W. K. (2014). Optimizing the growth performance of brown-marbled grouper, Epinephelus fuscoguttatus (Forskal), by varying the proportion of dietary protein and lipid levels. Anim. Feed Sci. Technol. 191, 98-105. doi: 10.1016/j.anifeedsci.2014.01.020

Susnik, S., Berrebi, P., Dovc, P., Hansen, M. M., and Snoj, A. (2004). Genetic introgression between wild and stocked salmonids and the prospects for using molecular markers in population rehabilitation: the case of the Adriatic grayling (Thymallus thymallus L. 1785). Heredity (Edinb). 93, 273-282. doi: 10.1038/sj. hdy. 6800500

Swain, P., Dash, S., Sahoo, P. K., Routray, P., Sahoo, S. K., Gupta, S. D., et al. (2007). Non-specific immune parameters of brood Indian major carp Labeo rohita and their seasonal variations. Fish Shellfish Immunol. 22, 38-43. doi: 10.1016/j.fsi.2006.03.010

Thomassen, M. S., Bou, M., Røsjø, C., and Ruyter, B. (2017). Organ and phospholipid class fatty acid specificity in response to dietary depletion of essential n-3 fatty acids in Atlantic salmon (Salmo salar L.). Aquac. Nutr. 23, 433-443. doi: 10.1111/anu.12409

Uiblein, F., Jagsch, A., Honsig-Erlenburg, W., and Weiss, S. (2001). Status, habitat use, and vulnerability of the European grayling in Austrian waters. J. Fish Biol. 59, 223-247. doi: 10.1006/jfbi.2001.1762

Walton, M. J. (1985). "Aspects of amino acid metabolism in teleost fish," in Nutrition and Feeding in Fish, eds C. V. Cowey, A. M. Mackie, and J. G. Bell (London: Academic Press).

Walton, M. J., and Cowey, C. B. (1982). Aspects of intermediary metabolism in salmonid fish. Comp. Biochem. Physiol. B Biochem. Mol. Biol. 73, 59-79. doi: 10.1016/0305-0491(82)90201-2

Wang, C., Hu, G., Sun, P., Gu, W., Wang, B., Xu, Q., et al. (2018). Effects of dietary protein at two lipid levels on growth, gonadal development, body composition and liver metabolic enzymes of brown trout (Salmo trutta fario) broodstock. Aquac. Nutr. 24, 1587-1598. doi: 10.1111/anu.12795

Wang, J. T., Han, T., Li, X. Y., Yang, Y. X., Yang, M., Hu, S. X., et al. (2017). Effects of dietary protein and lipid levels with different protein-to-energy ratios on growth performance, feed utilization and body composition of juvenile redspotted grouper, Epinephelus akaara. Aquac. Nutr. 23, 994-1002. doi: 10.1111/ anu. 12467

Wang, L., Hu, S., Lou, B., Chen, D., Zhan, W., Chen, R., et al. (2018). Effect of different dietary protein and lipid levels on the growth, body composition, and intestinal digestive enzyme activities of juvenile yellow drum Nibea albiflora (Richardson). J. Ocean Univ. China 17, 1261-1267. doi: 10.1007/s11802-0183660-1

Wang, L., Zhang, W., Gladstone, S., Ng, W. K., Zhang, J., and Shao, Q. (2019). Effects of isoenergetic diets with varying protein and lipid levels on the growth, feed utilization, metabolic enzymes activities, antioxidative status and serum biochemical parameters of black sea bream (Acanthopagrus schlegelii). Aquaculture 513:734397. doi: 10.1016/j.aquaculture.2019.734397

Webb, K. A., and Gatlin, D. M. (2003). Effects of dietary protein level and form on production characteristics and ammonia excretion of red drum Sciaenops ocellatus. Aquaculture 225, 17-26. doi: 10.1016/S0044-8486(03)00274-6 
Wu, X., and Gatlin, D. M. III (2014). Effects of altering dietary protein content in morning and evening feedings on growth and ammonia excretion of red drum (Sciaenops ocellatus). Aquaculture 434, 33-37. doi: 10.1016/j.aquaculture.2014. 07.019

Xu, G. F., Wang, Y. Y., Han, Y., Liu, Y., Yang, Y. H., Yu, S. L., et al. (2015). Growth, feed utilization and body composition of juvenile Manchurian trout, Brachymystax lenok (Pallas) fed different dietary protein and lipid levels. Aquac. Nutr. 21, 332-340. doi: 10.1111/anu.12165

Yamamoto, T., Unuma, T., and Akiyama, T. (2000). The influence of dietary protein and fat levels on tissue free amino acid levels of fingerling rainbow trout (Oncorhynchus mykiss). Aquaculture 182, 353-372. doi: 10.1016/S00448486(99)00277-X

Yang, S. D., Liou, C. H., and Liu, F. G. (2002). Effects of dietary protein level on growth performance, carcass composition and ammonia excretion in juvenile silver perch (Bidyanus bidyanus). Aquaculture 213, 363-372. doi: 10.1016/ S0044-8486(02)00120-5

Yin, P., Xie, S., Zhuang, Z., He, X., Tang, X., Tian, L., et al. (2021). Dietary supplementation of bile acid attenuate adverse effects of high-fat diet on growth performance, antioxidant ability, lipid accumulation and intestinal health in juvenile largemouth bass (Micropterus salmoides). Aquaculture 531:735864. doi: 10.1016/j.aquaculture.2020.735864

Żebrowska, E., Maciejczyk, M., Żendzian-Piotrowska, M., Zalewska, A., and Chabowski, A. (2019). High protein diet induces oxidative stress in rat cerebral cortex and hypothalamus. Int. J. Mol. Sci. 20:1547. doi: 10.3390/ijms20071547

Zhang, J., Zhang, X., Wang, H., Chen, L., Lin, B., Li, G., et al. (2018a). Effects of dietary protein and lipid levels on growth performance, feed utilization and biochemical parameters of barbless carp (Cyprinus pellegrini). ISR. J. Aquac. 70:9.

Zhang, J., Zhao, N., Sharawy, Z., Li, Y., Ma, J., and Lou, Y. (2018b). Effects of dietary lipid and protein levels on growth and physiological metabolism of Pelteobagrus fulvidraco larvae under recirculating aquaculture system (RAS). Aquaculture 495, 458-464. doi: 10.1016/j.aquaculture.2018.06.004

Zhang, J., Zhou, F., Wang, L., Shao, Q., Xu, Z., and Xu, J. (2010). Dietary protein requirement of juvenile black sea bream, Sparus macrocephalus. J. World Aquac. Soc. 41, 151-164. doi: 10.1111/j.1749-7345.2010.00356.x
Zhang, M., Chen, C., You, C., Chen, B., Wang, S., and Li, Y. (2019). Effects of different dietary ratios of docosahexaenoic to eicosapentaenoic acid (DHA/EPA) on the growth, non-specific immune indices, tissue fatty acid compositions and expression of genes related to LC-PUFA biosynthesis in juvenile golden pompano Trachin. Aquaculture 505, 488-495. doi: 10.1016/j. aquaculture.2019.01.061

Zhang, Y., Sun, Z., Wang, A., Ye, C., and Zhu, X. (2017). Effects of dietary protein and lipid levels on growth, body and plasma biochemical composition and selective gene expression in liver of hybrid snakehead (Channa maculata $q \times$ Channa argus $\sigma^{7}$ ) fingerlings. Aquaculture 468, 1-9. doi: 10.1016/j.aquaculture. 2016.09.052

Zuo, R., Ai, Q., Mai, K., Xu, W., Wang, J., Xu, H., et al. (2012). Effects of dietary docosahexaenoic to eicosapentaenoic acid ratio (DHA/EPA) on growth, nonspecific immunity, expression of some immune related genes and disease resistance of large yellow croaker (Larmichthys crocea) following natural infestation of paras. Aquaculture 334, 101-109. doi: 10.1016/j.aquaculture.2011. 12.045

Conflict of Interest: The authors declare that the research was conducted in the absence of any commercial or financial relationships that could be construed as a potential conflict of interest.

Publisher's Note: All claims expressed in this article are solely those of the authors and do not necessarily represent those of their affiliated organizations, or those of the publisher, the editors and the reviewers. Any product that may be evaluated in this article, or claim that may be made by its manufacturer, is not guaranteed or endorsed by the publisher.

Copyright (C) 2021 Rahimnejad, Dabrowski, Izquierdo, Malinovskyi, Kolárová and Policar. This is an open-access article distributed under the terms of the Creative Commons Attribution License (CC BY). The use, distribution or reproduction in other forums is permitted, provided the original author(s) and the copyright owner(s) are credited and that the original publication in this journal is cited, in accordance with accepted academic practice. No use, distribution or reproduction is permitted which does not comply with these terms. 